OPEN ACCESS

Edited by:

Chad Gregory Rose,

Auburn University, United States

Reviewed by: Nikunj Arunkumar Bhagat, University of Houston, United States

Eduardo Ros,

University of Granada, Spain

*Correspondence: Udeshika C. Dissanayake udeshikad@outlook.com

Received: 02 October 2021 Accepted: 20 December 2021 Published: 20 January 2022

Citation:

Dissanayake UC, Steuber V and Amirabdollahian F (2022) EEG

Spectral Feature Modulations Associated With Fatigue in Robot-Mediated Upper Limb Gross and Fine Motor Interactions. Front. Neurorobot. 15:788494. doi: 10.3389/fnbot.2021.788494

\section{EEG Spectral Feature Modulations Associated With Fatigue in Robot-Mediated Upper Limb Gross and Fine Motor Interactions}

\author{
Udeshika C. Dissanayake*, Volker Steuber and Farshid Amirabdollahian \\ School of Physics, Engineering and Computer Science, University of Hertfordshire, Hatfield, United Kingdom
}

This paper investigates the EEG spectral feature modulations associated with fatigue induced by robot-mediated upper limb gross and fine motor interactions. Twenty healthy participants were randomly assigned to perform a gross motor interaction with HapticMASTER or a fine motor interaction with SCRIPT passive orthosis for 20 min or until volitional fatigue. Relative and ratio band power measures were estimated from the EEG data recorded before and after the robot-mediated interactions. Paired-samples $t$-tests found a significant increase in the relative alpha band power and a significant decrease in the relative delta band power due to the fatigue induced by the robot-mediated gross and fine motor interactions. The gross motor task also significantly increased the $(\theta+\alpha) / \beta$ and $\alpha / \beta$ ratio band power measures, whereas the fine motor task increased the relative theta band power. Furthermore, the robotmediated gross movements mostly changed the EEG activity around the central and parietal brain regions, whereas the fine movements mostly changed the EEG activity around the frontopolar and central brain regions. The subjective ratings suggest that the gross motor task may have induced physical fatigue, whereas the fine motor task may have induced mental fatigue. Therefore, findings affirm that changes to localised brain activity patterns indicate fatigue developed from the robot-mediated interactions. It can also be concluded that the regional differences in the prominent EEG spectral features are most likely due to the differences in the nature of the task (fine/gross motor and distal/proximal upper limb) that may have differently altered an individual's physical and mental fatigue level. The findings could potentially be used in future to detect and moderate fatigue during robot-mediated post-stroke therapies.

Keywords: electroencephalogram, fatigue in upper limb robot-mediated interactions, HapticMASTER, SCRIPT passive orthosis, relative band power, band power ratios, independent component analysis, statistical analysis

\section{INTRODUCTION}

Fatigue experienced during post-stroke upper limb rehabilitation and its implications for the therapy outcome are often overlooked in existing therapy sessions. Many stroke survivors (about 30 to $70 \%$ ) have reported persistence of fatigue as a debilitating symptom (Staub and Bogousslavsky, 2001; Lerdal et al., 2009). It is more likely that the increased motor/cognitive processing demands required during motor retraining exercises may exacerbate stroke patients' fatigue levels. The 
elevated fatigue levels may impair motivation and compliance to effectively perform the therapeutic interactions and the long-term commitment toward rehabilitation. Furthermore, some studies have reported that high-intensity fatiguing tasks are detrimental to both motor performance and learning (Godwin and Schmidt, 1971; Carron, 1972; Thomas et al., 1975; Williams and Singer, 1975; Branscheidt et al., 2019), whereas some investigations have only found performance impairments (Alderman, 1965; Carron, 1969; Cotten et al., 1972). Sterr and Furlan (2015) hypothesised that the relationship between training intensity and motor performance of constraint-induced therapy in chronic hemiparetic stroke patients is modulated by fatigue in addition to the residual motor ability. Foong et al. (2019) also suggested that the poor performance in the nBETTER (Neurostyle Brain Exercise Therapy Towards Enhanced Recovery) system could be due to the mental fatigue that progressed during the therapy. In Prasad's et al. (2010) study where chronic hemiplegic stroke patients performed both physical practice and motor imagery, a trend of more considerable variability in the brain-computer interface (BCI) performance was observed with the rise in individual fatigue levels. Therefore, it is highly questionable whether continuing a stroke therapy while or beyond fatigued conditions would impede motor performance and motor skill relearning during therapeutic interactions.

Despite its clinical importance, there exists no unambiguous and universally agreed definition for the term fatigue. In general, fatigue is a sensation of tiredness, weariness or lack of energy that is experienced following or during prolonged physical or mental activity. Fatigue can be broadly categorised into two types: physical (or muscular) fatigue and mental fatigue. Physical fatigue is defined as a failure to maintain force (or power output) during sustained muscle contractions (Gibson and Edwards, 1985). In contrast, mental fatigue is a subjective feeling of tiredness experienced during or after prolonged periods of demanding cognitive activity (Lorist et al., 2005). Recent studies have also shown that mental fatigue impairs physical performance, especially in sports-related activities (Marcora et al., 2009; Mehta and Parasuraman, 2014; Van Cutsem et al., 2017). Electroencephalogram (EEG) has shown to be the most predictive and promising biomarker of fatigue (Lal and Craig, 2001; Tran et al., 2020). To date, many studies have investigated EEG feature modulations associated with fatigue, including fatigue induced by driving tasks (Lal and Craig, 2002; Eoh et al., 2005; Tran et al., 2008; Jap et al., 2009; Craig et al., 2012; Zhao et al., 2012; Borghini et al., 2014), voluntary motor tasks (Yao et al., 2009; Wang et al., 2017), cognitive tasks (Massar et al., 2010; Tanaka et al., 2012; Trejo et al., 2015), brain-computer interfaces (Käthner et al., 2014), exercises and sports-related activities (Bailey et al., 2008; Barwick et al., 2012; Baumeister et al., 2012; Xu et al., 2018), visual display terminal tasks (Cheng and Hsu, 2011; Fan et al., 2015). However, the alterations in EEG activity caused by fatigue accumulated following robot-mediated interactions have not yet been comprehensively explored to the

Abbreviations: $\alpha_{\text {relative, }}$, relative alpha band power; $\beta_{\text {relative, }}$, relative beta band power; $\delta_{\text {relative }}$, relative delta band power; $\theta_{\text {relative }}$, relative theta band power. author's knowledge. EEG-based fatigue indices could be used to mitigate fatigue accumulated during human-robot interactions, thereby enhancing the efficacy of rehabilitation and reducing fatigue-related risks in human-robot collaboration tasks.

EEG consists of a wide frequency spectrum, and spectral features (band power and band power ratios) are frequently used as indicators of fatigue. Table $\mathbf{1}$ summarises the findings of 16 studies over the last two decades identified by a systematic review on EEG spectral feature modulations caused by fatigue. It was evident that in most studies, $\theta$ and $\alpha$ band power increased and $\beta$ band power decreased significantly as a result of fatigue (Lal and Craig, 2002; Eoh et al., 2005; Barwick et al., 2012; Craig et al., 2012; Zhao et al., 2012; Käthner et al., 2014; Fan et al., 2015; Trejo et al., 2015; Zou et al., 2015; Wang et al., 2017; Xu et al., 2018). Some studies investigated the variations in delta band power as well; however, not many studies were able to identify significant variations with fatigue (Caldwell et al., 2002; Lal and Craig, 2002; Jap et al., 2009; Craig et al., 2012; Tanaka et al., 2012; Zhao et al., 2012; Chen et al., 2013; Fan et al., 2015). In these studies, EEG band power is given as absolute band power or relative band power. The relative band power is defined as a ratio between the absolute band power of each frequency band and the total power of all frequency bands in consideration. EEG band power ratios: $(\theta+\alpha) / \beta, \alpha / \beta,(\theta+\alpha) /(\alpha+\beta)$, and $\theta / \beta$ were also used in some studies since the basic band powers can be insufficient to observe the shift of brain activity from fast waves to slow waves (Eoh et al., 2005; Jap et al., 2009; Fan et al., 2015). EEG band power ratios showed a significant increase with fatigue build-up. Eoh et al. (2005) stated that the index $(\theta+\alpha) / \beta$ was a more reliable fatigue indicator during a simulated driving task due to the mutual addition of $\alpha$ and $\theta$ activity during the repetitive phase transition between wakefulness and microsleep. Jap et al. (2009) also reported a greater increase in the index $(\theta+\alpha) / \beta$, in comparison to the other power ratios, when a person experienced a fatigued state at the end of a monotonous simulated driving task. Most studies have also found a widespread topographical distribution in the changes in EEG spectral features with fatigue. However, some studies are equivocal and need further exploration (Jap et al., 2009; Cheng and Hsu, 2011; Baumeister et al., 2012; Tanaka et al., 2012; Chen et al., 2013). Variations in methodological approaches, including low sample size, differences in the fatiguing study protocol, the number of electrodes used, the electrode placement and the feature definition, could be a possible explanation for the discrepancies across the studies.

The type of fatigue experienced during robot-mediated exercises may depend on the exercise mode, intensity and condition of the patient. For instance, the upper limb joints and muscles involved in an interaction may vary from one therapy to another depending on the severity of the impairment of fine or gross motor skills. Gross motor skill retraining exercises such as arm reach/return exercises are primarily involved in the movement and coordination of proximal joints and muscles of the upper limb (shoulder and arm). In contrast, fine motor skill retraining exercises involve coordination of the distal joints and muscles of the upper limb (hand, wrist, and fingers). Cowley and Gates (2017) found that proximal fatigue in a repetitive, 
TABLE 1 | Literature summary on modulations in the EEG spectral features with fatigue.

\begin{tabular}{|c|c|c|c|c|c|c|c|c|c|c|c|c|}
\hline Reference & Description & $\begin{array}{c}\text { No of } \\
\text { participants }\end{array}$ & $\begin{array}{l}\text { No of } \\
\text { electrodes }\end{array}$ & $\delta$ & $\theta$ & $\alpha$ & $\beta$ & $\frac{(\theta+\alpha)}{\beta}$ & $\frac{\alpha}{\beta}$ & $\frac{(\theta+\alpha)}{(\alpha+\beta)}$ & $\frac{\theta}{\beta}$ & $\begin{array}{l}\text { Electrode locations or } \\
\text { brain regions modulated } \\
\text { by fatigue }\end{array}$ \\
\hline $\begin{array}{l}\text { Barwick et al. } \\
\text { (2012) }\end{array}$ & $\begin{array}{l}\text { Fatigue during } \\
\text { administration of a } \\
\text { neuropsychological test } \\
\text { battery }\end{array}$ & 14 & 42 & - & $\uparrow^{R}$ & $\uparrow^{R}$ & $\downarrow^{R}$ & - & - & - & - & $\mathrm{F}, \mathrm{C}, \mathrm{P}, \mathrm{O}$ \\
\hline $\begin{array}{l}\text { Baumeister et al. } \\
\text { (2012) }\end{array}$ & $\begin{array}{l}\text { Effects of fatigue induced by } \\
\text { a cycling exercise on knee } \\
\text { joint reproduction task }\end{array}$ & 12 & 22 & - & $\downarrow$ & $\downarrow^{L, U}$ & - & - & - & - & - & $\begin{array}{l}\text { F3, Fz, F4, FC3, FCz, FC4, } \\
\text { P4, O1, Oz, O2, T5 }\end{array}$ \\
\hline Chen et al. (2013) & $\begin{array}{l}\text { Fatigue induced by } \\
\text { watching 3DTV }\end{array}$ & 10 & 16 & $\uparrow^{R}$ & NS & $\downarrow^{R}$ & $\downarrow^{R}$ & $\uparrow$ & $\uparrow$ & $\uparrow$ & $\uparrow$ & $\begin{array}{l}\text { FP1, FP2, F3, C3, C4, F7, } \\
\text { F8, T5 }\end{array}$ \\
\hline $\begin{array}{l}\text { Cheng and Hsu } \\
\text { (2011) }\end{array}$ & $\begin{array}{l}\text { Mental fatigue induced by } \\
\text { visual display terminal tasks }\end{array}$ & 20 & 7 & - & $\uparrow^{R}$ & $\downarrow^{R}$ & NS & $\downarrow$ & $N S^{a}$ & - & - & $\mathrm{F} 3, \mathrm{Fz}, \mathrm{F} 4, \mathrm{Cz}, \mathrm{Pz}, \mathrm{O} 1, \mathrm{O} 2$ \\
\hline Craig et al. (2012) & $\begin{array}{l}\text { Fatigue induced by } \\
\text { monotonous simulated } \\
\text { driving task }\end{array}$ & 48 & 32 & NS & $\uparrow$ & $\uparrow^{L, U}$ & $\uparrow$ & - & - & - & - & $\begin{array}{l}\text { FL, FM, FR, CL, CM, CR, } \\
\text { POL, POM, POR }\end{array}$ \\
\hline Fan et al. (2015) & $\begin{array}{l}\text { Mental fatigue in visual } \\
\text { search task }\end{array}$ & 10 & 64 & NS & NS & $\uparrow^{R}$ & $\downarrow^{R}$ & $\uparrow$ & $\uparrow$ & $\uparrow$ & $\uparrow$ & FP, IF, F, C, P, O, T, PT \\
\hline Jap et al. (2009) & $\begin{array}{l}\text { Fatigue induced during a } \\
\text { monotonous driving session }\end{array}$ & 52 & 30 & * & $*$ & $\downarrow$ & $\downarrow$ & $\uparrow$ & $\uparrow$ & $\uparrow$ & $\uparrow$ & $\mathrm{F}, \mathrm{C}, \mathrm{P}, \mathrm{T}, \mathrm{EB}$ \\
\hline $\begin{array}{l}\text { Käthner et al. } \\
\text { (2014) }\end{array}$ & $\begin{array}{l}\text { Mental fatigue during P300 } \\
\text { brain computer interface }\end{array}$ & 12 & 31 & - & $\uparrow$ & $\uparrow$ & - & - & - & - & - & $\begin{array}{l}\text { F3, Fz, F4, FC5, FC3, FCz, } \\
\text { FC4, FC6, C5, C3, Cz, C4, } \\
\text { C6, CP5, CP3, CPz, CP4, } \\
\text { CP6, P3, P1, Pz, P2, } \\
\text { P4,PO7, PO3, POz, PO4, } \\
\text { PO8, O1, O2 }\end{array}$ \\
\hline $\begin{array}{l}\text { Lal and Craig } \\
\text { (2002) }\end{array}$ & $\begin{array}{l}\text { Fatigue during simulated } \\
\text { driving task }\end{array}$ & 35 & 19 & $\uparrow$ & $\uparrow$ & $\uparrow$ & $\uparrow$ & - & - & - & - & $\mathrm{EB}$ \\
\hline Xu et al. (2018) & $\begin{array}{l}\text { Fatigue in mental(NS) and } \\
\text { physical-mental task }\end{array}$ & 14 & 16 & - & - & - & $\downarrow^{R}$ & - & $\uparrow$ & - & - & C3, P3, Pz, Oz, T3, T4, T5 \\
\hline Zhao et al. (2012) & $\begin{array}{l}\text { Mental fatigue in simulated } \\
\text { driving task }\end{array}$ & 13 & 32 & NS & $\uparrow^{R}$ & $\uparrow^{R}$ & $\downarrow^{R}$ & - & - & - & - & $\mathrm{F}, \mathrm{C}, \mathrm{P}, \mathrm{O}, \mathrm{T}$ \\
\hline Zou et al. (2015) & $\begin{array}{l}\text { Stereoscopic 3D visual } \\
\text { fatigue caused by } \\
\text { vergence-accommodation } \\
\text { conflict }\end{array}$ & 11 & 30 & - & NS & $\uparrow^{R}$ & $\downarrow^{R}$ & * & $*$ & NS & NS & $F, C, P, E B$ \\
\hline
\end{tabular}

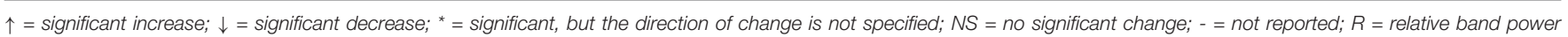

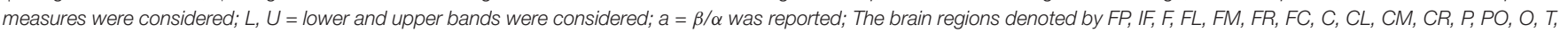

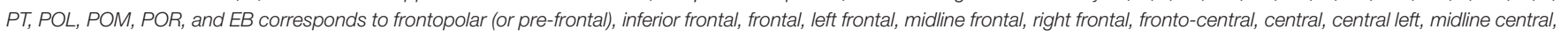
central right, parietal, parieto-occipital, occipital, temporal, posterior temporal, posterior left, midline posterior, posterior right and entire brain average.

timed movement task significantly changes the movement in trunk shoulder and elbow kinematics, whereas the changes were mainly in wrist and hand movement due to distal muscle fatigue. Therefore, in general, repetitive gross motor skill retraining exercises may induce more physical fatigue than fine motor skill retraining exercises. In addition, most therapeutic fine motor activities require considerable attention and decision-making skills combined with hand, wrist and finger movements; therefore, they may induce more mental fatigue than most gross motor exercises. As the type of prominent fatigue developed during a robot-mediated interaction may vary depending on the physical and mental workload associated with the task, cortical 

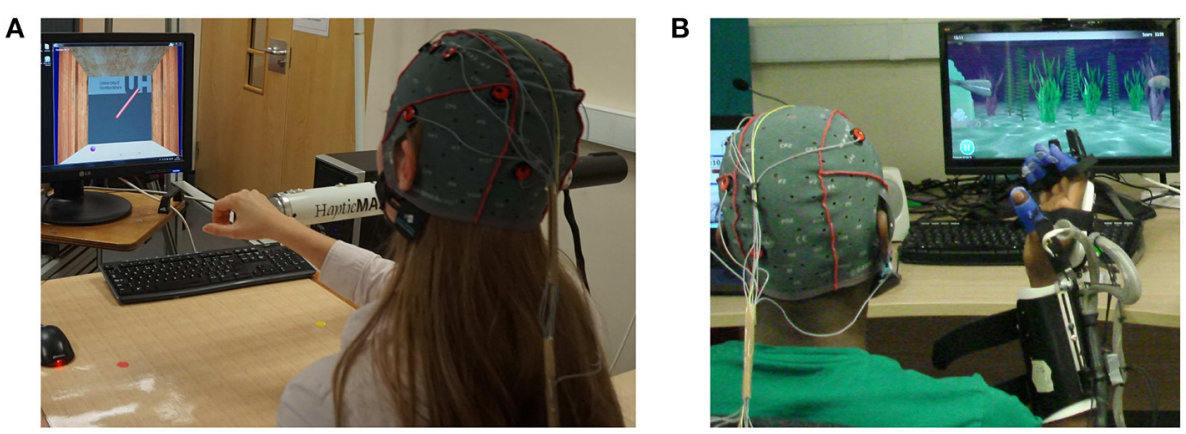

FIGURE 1 | Fatigue inducing robot-mediated interactions. (A) Robot-mediated gross motor interaction (arm reach/return task) using HapticMASTER, and (B) robot-mediated fine motor interaction (hand open/close task) using SCRIPT passive orthosis.

sites that show significant variations in EEG spectral features following fatigue may differ between interactions. However, these differences between gross and fine motor robot-mediated interactions are not systematically investigated.

In this preliminary experiment, we hypothesised that the EEG correlates of fatigue induced by robot-mediated interactions are specific to the physical or cognitive nature of the task and the differences in the usage of the proximal or distal upper limb. The gross movements (arm reach/return) were performed using the HapticMASTER (Motekforce Link, The Netherland) (Amirabdollahian et al., 2007; Chemuturi et al., 2013), and the fine movements (hand open/close) were performed using the SCRIPT passive orthosis (Amirabdollahian et al., 2014). Given the differences in the two tasks, it could be expected that the gross motor task may induce more physical fatigue than the fine motor task, in which more mental fatigue may be visible. Therefore, it was anticipated that the resulting statistically significant differences in EEG spectral features might show varying topographical distributions between the two robotmediated interactions. Furthermore, significant changes to the EEG spectral features localised around the motor cortex were expected following the robot-mediated gross movements since fatigue may affect motor coordination skills. In the fine motor robot-mediated interaction that requires more attention and decision making, significant changes to the frontopolar brain activities were expected in addition to the attenuation in the activities around the motor cortex.

\section{MATERIALS AND METHODS}

\subsection{Ethical Approval}

The experiment was approved by the Science and Technology Ethics Committee with Delegated Authority of the University of Hertfordshire (Protocol numbers: COM/PG/UH/00100 and aCOM/PG/UH/00100).

\subsection{Participants}

Twenty healthy right-handed volunteers, who were at least 20 years of age (average age of the sample was $32 \pm 10$ years; mean $\pm \mathrm{SD}$ ) and with no history of severe injury to the head, brain, or right hand participated in this experiment. Right-handedness was considered since both robotic interfaces were constrained to be used only by the right upper limb due to their hardware configurations and setup. All participants had normal vision or corrected to normal vision. All participants signed informed consent forms before participation.

\subsection{Fatigue Inducing Robot-Mediated Interactions}

Given the consent to take part in the experiment, participants were randomly assigned into two groups: $\mathrm{A}$ and $\mathrm{B}$, with 10 participants in each group. Participants in group A performed visually guided arm reach/return movements with HapticMASTER (gross motor task, Figure 1A), whereas participants in group B performed hand open/close movements with SCRIPT passive orthosis (fine motor task, Figure 1B). Both robot-mediated interactions were performed for 20-min or until volitional fatigue. The virtual reality environment of the GENTLE/A rehabilitation system (Chemuturi et al., 2013) was used for the gross motor task. Target point locations were modified so that the trajectory covered by the movement of the HapticMASTER robot arm was mapped into a straight line connecting only two virtual target points. In addition, the HapticMASTER was set to active mode so that the participants should initiate the movement and reach the target points by themselves. The virtual reality game "sea shell," developed for the SCRIPT system, was used as the fine motor task (Amirabdollahian et al., 2014). Participants performed hand open/close gestures to open/close a seashell underwater to catch a fish near the seashell. Both robot-mediated interactions were performed using only the right hand, and participants were asked to keep their left hand in a relaxed position throughout the task. The distance between the computer monitor and the participant's eye was set to around $120 \mathrm{~cm}$ for both groups.

\subsection{EEG Data Acquisition}

Continuous EEG signals were recorded before, during and after the robotic interactions using an eight-channel EEG data acquisition system, g.MOBIlab+ (g.tec medical engineering $\mathrm{GmbH}$, Austria) with active electrodes. According to the International 10-10 system of electrode placement (American, 2006), FP1, F3, FC3, C3, C4, P3, O1, and T7 electrode 


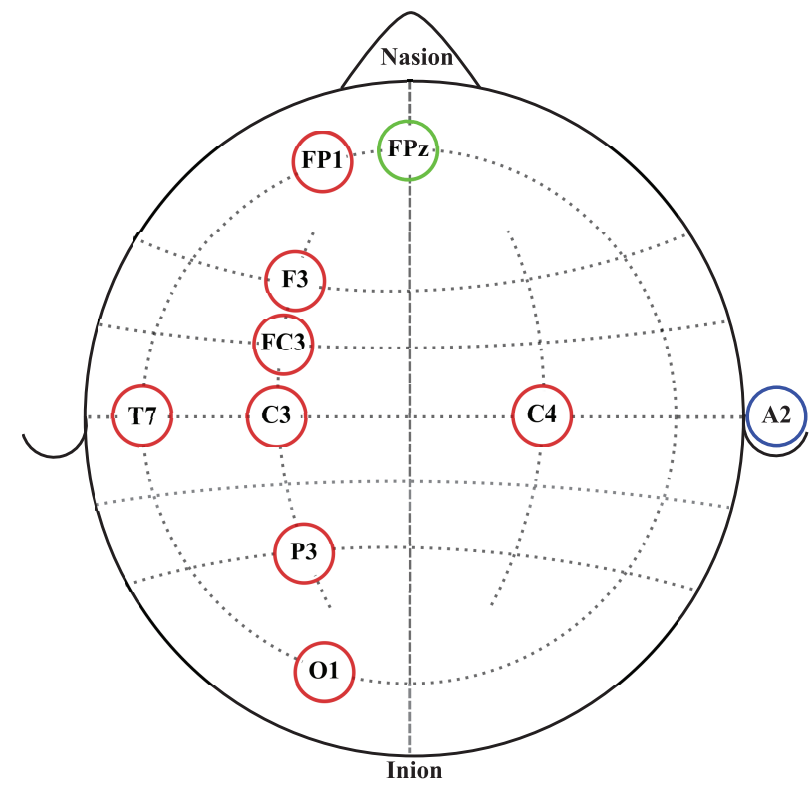

FIGURE 2 | EEG electrode placement according to the International 10-10 system of electrode placement. Red circles represent the eight active electrodes selected for the data acquisition. The blue circle represents the reference electrode location. The green circle represents the ground electrode location.

locations were selected as shown in Figure 2. All electrodes were referenced to the right earlobe (A2), and $\mathrm{FPz}$ was used as the ground electrode. Signals acquired by the active electrodes are pre-amplified directly at the electrode (Pinegger et al., 2016). Also, the active electrode system reduces or avoids artifacts caused by high impedance between the electrode(s) and the skin (e.g., 50/60 Hz coupling, electrode or cable movement artifacts, background noise) (g.tec medical engineering $\mathrm{GmbH}, 2014 \mathrm{~b}$ ). The sampling rate, lower and upper cut-off frequencies of the bandpass filter of the amplifier are fixed at 256, 0.5, and 100 $\mathrm{Hz}$, respectively, by the manufacturer. Therefore, signals acquired from this device were sampled at $256 \mathrm{~Hz}$ and had a fixed EEG bandwidth of 0.5 to $100 \mathrm{~Hz}$.

\subsection{Experimental Procedure}

On arrival at the laboratory, participants were informed about the experiment protocol, given time to familiarise themselves with the assigned robotic interaction and were prepared for the EEG data collection according to the guidelines given in g.tec medical engineering $\mathrm{GmbH}$ (2014a). The flow diagram of the proposed experiment is given in Figure 3. Following the standardised EEG recording protocol, EEG data were recorded before, during and after the robot-mediated interactions. Participants were instructed to close and open their eyes for $180 \mathrm{~s}$ each when EEG data were recorded before and after the gross and fine motor tasks. In order to reduce artifacts in the EEG data recorded with eyes opened/closed, participants were instructed to sit still while minimising eye blinks, eye movements, swallowing, jaw clenching, or any other severe body movements.
In this paper, only the EEG data recorded with eyes opened are further analysed. Participant's feedback on their physical and mental fatigue level before and after the tasks were obtained using two statements with a 5-point Likert rating scale (i.e., $1=$ "Not at all fatigued," 2 = "somewhat fatigued," 3 = "moderately fatigued," 4 = "very fatigued," and 5 = "extremely fatigued"). Also, the participant's feedback on the task-associated physical and mental workload was obtained using two statements with a 5-point Likert rating scale (i.e., 1 = "Not at all demanding", 2 = "somewhat demanding," 3 = "moderately demanding," 4 = "very demanding," and 5 = "extremely demanding") (Dissanayake, 2021). Moreover, all participants performed the assigned task for $20 \mathrm{~min}$.

\subsection{EEG Data Analysis}

This paper reports the modulation of EEG spectral features during eyes opened states before and after the fatiguing robotmediated interactions. EEG features extracted from the data recorded before the task is referred to as baseline, and the data recorded after the task is referred to as recovery, respectively, throughout this paper. These states can be considered to reflect the restfulness of the participant before and after the robotic interactions; thereby, any changes in these states could be a reflection of fatigue. Previous studies have also compared EEG data recorded before and after a task to identify EEG feature modulations associated with fatigue induced by physical and mental tasks (Ng and Raveendran, 2007; Cheng and Hsu, 2011; Tanaka et al., 2012; Chen et al., 2013). The EEG data processing pipeline followed for each participant during baseline and recovery states is illustrated in Figure 4. EEG preprocessing and feature extraction was performed offline using custom MATLAB scripts.

\section{Preprocessing}

Firstly, the DC offset of each recording was removed by subtracting the channel-wise mean from each data point. Then, a Type II Chebyshev low-pass filter with a stopband frequency of $45 \mathrm{~Hz}$ and an order of 20 was applied to eliminate the power line noise $(50 \mathrm{~Hz})$ distortions.

The EEG research community widely uses independent component analysis (ICA) to separate and remove artifacts in EEG signals (Makeig et al., 1996; Jung et al., 1998; Delorme et al., 2007; Debener et al., 2010). ICA is a linear decomposition technique used to recover a set of $n$ unobserved independent source signals given only $m \geq n$ observed instantaneous mixtures of these source signals. If we denote the $n$ independent source signals at time $t$ by a $n \times 1$ vector $\mathbf{s}(t)$ and the observed signals by a $m \times 1$ vector $\mathbf{x}(t)$, the mixing model can be written as,

$$
\mathbf{x}(t)=\operatorname{As}(t),
$$

where the $m \times n$ matrix $\mathbf{A}$ represents the unknown "mixing matrix". The elements in each row of A corresponds to the contributions from each source signal to each observation (i.e., $x_{i}(t)=\sum_{j=1}^{n} a_{i j} s_{j}(t)$ for all $i=1$ to $\left.m\right)$. The objective of ICA is to find a separating matrix, i.e., a $n \times m$ matrix $\mathbf{W}$ such that

$$
\mathbf{u}(t)=\mathbf{W} \mathbf{x}(t)
$$




\begin{tabular}{|c|c|c|c|c|}
\hline $\begin{array}{c}\text { Questionnaire on } \\
\text { participant } \\
\text { demography and }\end{array}$ & $\begin{array}{l}\text { Baseline EEG data } \\
\text { recording } \\
\text { - Eyes closed for } 180 \mathrm{~s}\end{array}$ & $\begin{array}{c}\text { Robot-mediated interaction } \\
\text { for } 20 \text { minutes or until } \\
\text { volitional fatigue and }\end{array}$ & $\begin{array}{l}\text { Recovery EEG data } \\
\text { recording } \\
\text { - Eyes closed for } 180 \mathrm{~s} \\
\end{array}$ & $\begin{array}{c}\text { Questionnaire on } \\
\text { post-task fatigue level, } \\
\text { workload and }\end{array}$ \\
\hline
\end{tabular}

FIGURE 3 | Flow diagram of the proposed experiment.

\section{Raw EEG}

(States: eyes opened before (baseline) and after (recovery) the robot-mediated interactions, duration: $180 \mathrm{~s}$ )

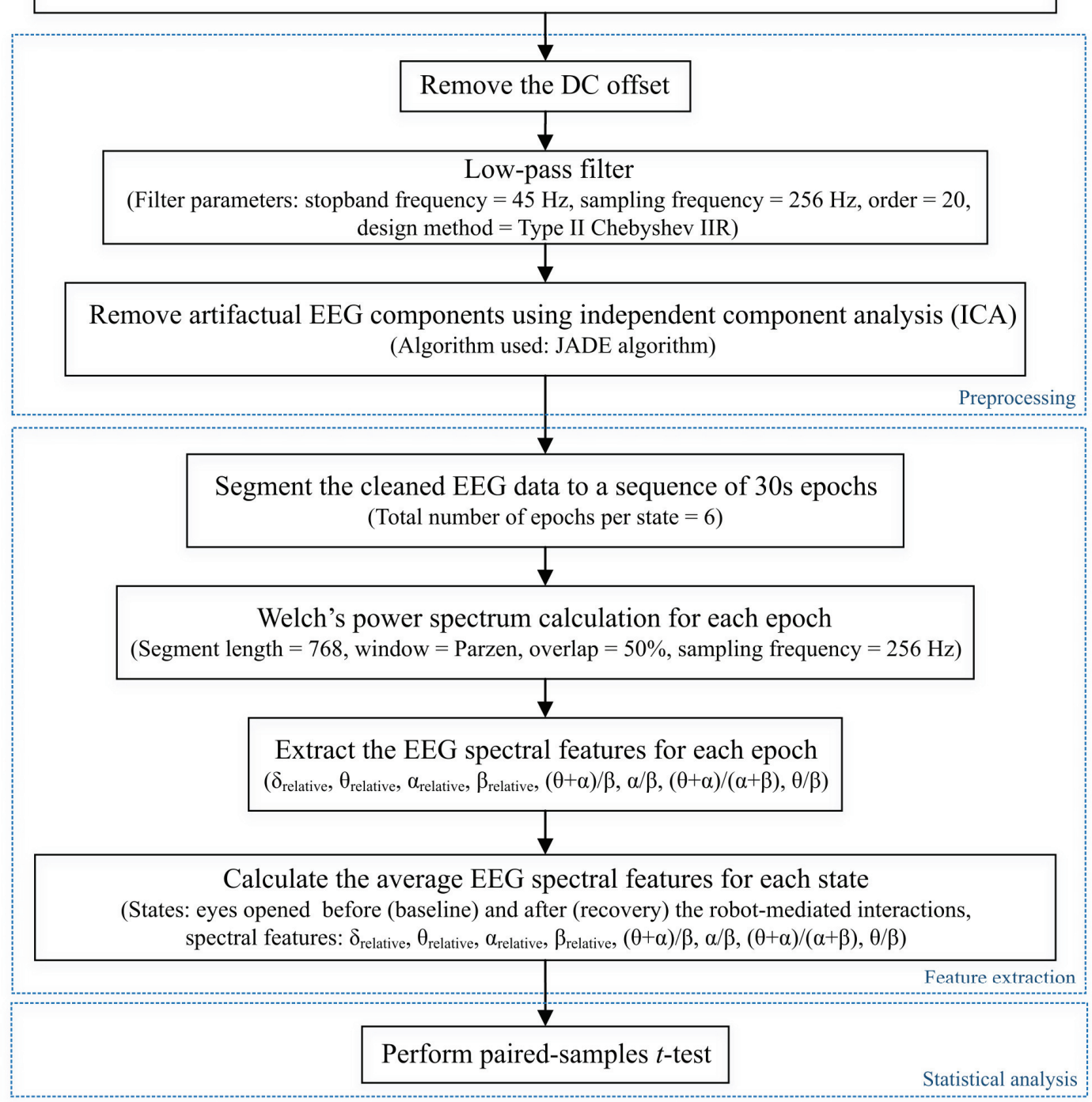

FIGURE 4 | EEG data processing pipeline followed to preprocess raw EEG data and extract EEG spectral features of each state for each participant to perform the statistical analysis. Dotted boxes represent the three main steps involved in the pipeline: data preprocessing, feature extraction, and statistical analysis. $\delta_{\text {relative }}, \theta_{\text {relative }}$, $\alpha_{\text {relative }}$, and $\beta_{\text {relative }}$ indicate the relative $\delta, \theta, \alpha$ and $\beta$ band powers, respectively, and $(\theta+\alpha) / \beta, \alpha / \beta,(\theta+\alpha) /(\alpha+\beta)$, and $\theta / \beta$ indicate the power ratios. 
is an estimate of the original source signals. The elements in the $n \times 1$ vector $\mathbf{u}(t)$ (i.e., independent components) are identical to the original source signals up to permutations and changes of scales and signs (Cardoso, 1998).

The joint approximate diagonalisation of eigenmatrices (JADE) algorithm (Cardoso and Souloumiac, 1993) was used in this experiment to separate and remove in-band artifacts, including eye blinking, eye movement, swallowing, jaw clenching, and cardiac activity from the independent components. Figure 5 illustrates the artifact removal process using ICA for a single subject. When applying ICA to separate EEG artifacts from brain activity patterns, it was assumed that the signals emitted by the unobserved sources are independent, and the number of independent sources is the same as the number of electrodes used in the experiment (i.e., $m=n=8$ ). The relative projection strengths of each independent component onto the scalp electrodes were given by the columns of the inverse separation matrix $\mathbf{W}^{-1}$, which is an estimate of the mixing matrix A in Equation 1. The "corrected" EEG signal was then derived as, $\hat{\mathbf{x}}(t)=W^{-1} \hat{\mathbf{u}}(t)$, where $\hat{\mathbf{u}}(t)$ was derived from the matrix of activation waveforms $\mathbf{u}(t)$, by setting the rows representing the artifactual components identified by visual inspection to zero (Jung et al., 2000).

\section{Feature Extraction}

The corrected EEG signals at the two states: baseline and recovery for each participant were segmented into epochs of $30 \mathrm{~s}$ length (i.e., 7680 samples per epoch and 6 epochs in total per state). The power spectral density for all epochs was estimated using Welch's averaged modified periodogram method (Welch, 1967) with a 3 s segment length (i.e., 768 samples), 50\% overlap, and a Parzen window. Subsequently, the relative band power of $\delta$ $(1-<4 \mathrm{~Hz}), \theta(4-<8 \mathrm{~Hz}), \alpha(8-13 \mathrm{~Hz})$, and $\beta(<13-30 \mathrm{~Hz})$ (denoted by $\delta_{\text {relative }}, \theta_{\text {relative }}, \alpha_{\text {relative }}$, and $\beta_{\text {relative }}$, respectively, in this paper) for each epoch were calculated as a ratio between the average band power of each frequency band and the total band power (i.e., the summation of average $\delta, \theta, \alpha$, and $\beta$ band powers). The four ratio band power measures: $(\theta+\alpha) / \beta, \alpha / \beta$, $(\theta+\alpha) /(\alpha+\beta)$, and $\theta / \beta$ for each epoch were also calculated. Finally, the average of each EEG spectral feature within the $180 \mathrm{~s}$ duration (i.e., six epochs) of each state was calculated to represent the corresponding spectral EEG feature index of the baseline and recovery states, respectively (Dissanayake, 2021).

\section{Statistical Analysis}

Statistical analysis was carried out using the IBM SPSS Statistics 25 software. A $p$-value $<0.05$ was considered statistically significant, denoting a $95 \%$ confidence interval. It was of interest to investigate whether the significant differences in EEG spectral features caused by fatigue are localised to different electrode locations due to the differences in the nature of the task (fine/gross motor and distal/proximal upper limb). Normality of the differences between EEG spectral features extracted from baseline and recovery states were assessed using the KolmogorovSmirnov test. Upon confirmation of normal distribution, twotailed paired-samples $t$-tests were performed separately on the eight electrode locations to identify the significant differences between the baseline and recovery states of each EEG spectral feature for each robot-mediated interaction. The effect sizes were expressed by the Pearsons' correlation coefficient, $r=\sqrt{\frac{t^{2}}{\left(t^{2}+d f^{2}\right)}}$. Multiple paired-samples $t$-tests were also used in previous fatigue studies to evaluate the changes in EEG features at different brain regions (Tanaka et al., 2012; Zhao et al., 2012; Chen et al., 2013; Fan et al., 2015).

\section{RESULTS}

\subsection{Modulations in EEG Spectral Features Following the Robot-Mediated Gross Motor Interaction With HapticMASTER}

Table 2 summarises the paired-samples $t$-test results of the statistically significant EEG spectral feature modulations following the gross motor interaction with HapticMASTER. Figure 6 shows the sample mean and standard deviation of the substantive EEG spectral features during baseline and recovery states. Comparison of the sub-figures shows that $\alpha_{\text {relative }}$ changed the most due to fatigue induced by the gross motor interaction with HapticMASTER. In Figure 6B, there is a clear increase in the sample mean of $\alpha_{\text {relative }}$ across all electrodes, with statistically significant differences visible on the three electrodes placed over the contralateral motor cortex: FC3 $(t(9)=-2.378, p=0.041, r$ $=0.621), \mathrm{C} 3(t(9)=-3.148, p=0.012, r=0.724)$ and $\mathrm{P} 3(t(9)$ $=-2.646, p=0.027, r=0.661)$. As well as being statistically significant, the effect of the variation in $\alpha_{\text {relative }}$ on FC3, C3, and P3 electrodes is large. These electrodes correspond to motor activities using the right hand; thereby, the significant increase in $\alpha_{\text {relative }}$ reflects a decreased cortical activation, which is an indication of fatigue. Similarly, Figures 6C,D show that fatigue induced by the gross motor task significantly increased both $(\theta+\alpha) / \beta(t(9)=-2.787, p=0.021, r=0.681)$ and $\alpha / \beta(t(9)=-$ 2.403, $p=0.040, r=0.625)$ on the $\mathrm{C} 3$ electrode. A larger effect size was also visible on the $\mathrm{C} 3$ electrode for both $(\theta+\alpha) / \beta$ and $\alpha / \beta$. These findings show that fatigue induced by gross movements increased the low-frequency power and decreased the fast wave activities on the $\mathrm{C} 3$ electrode, resulting in a significant difference when combined. In contrast, Figure $\mathbf{6 A}$ indicates that there has

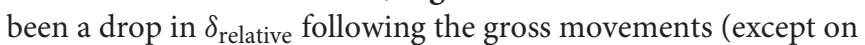
T7). Also, a significant variation with larger effect was found on the C3 electrode $(t(9)=2.593, p=0.029, r=0.654)$. This result is somewhat counter-intuitive because previous studies have shown a significant increase or no change in delta activity as fatigue progressed; however, it is reasonable to assume that this inconsistency may be related to the differences in experimental protocols. There were no significant differences visible in $\theta_{\text {relative }}$, $\beta_{\text {relative, }}(\theta+\alpha) /(\alpha+\beta)$, and $\theta / \beta$ due to fatigue induced by the gross motor task. Overall, these results show a reduced activation around the sensorimotor cortex due to fatigue induced by robot-mediated gross movements. Figure 7 shows the brain topographies of the difference between recovery and baseline states (i.e., difference $=$ recovery - baseline) of $\delta_{\text {relative }}, \alpha_{\text {relative }}$, $(\theta+\alpha) / \beta$ and $\alpha / \beta$ for one participant who reported a greater increase in physical fatigue than mental fatigue following the gross motor task. Topographical distributions also show that the 


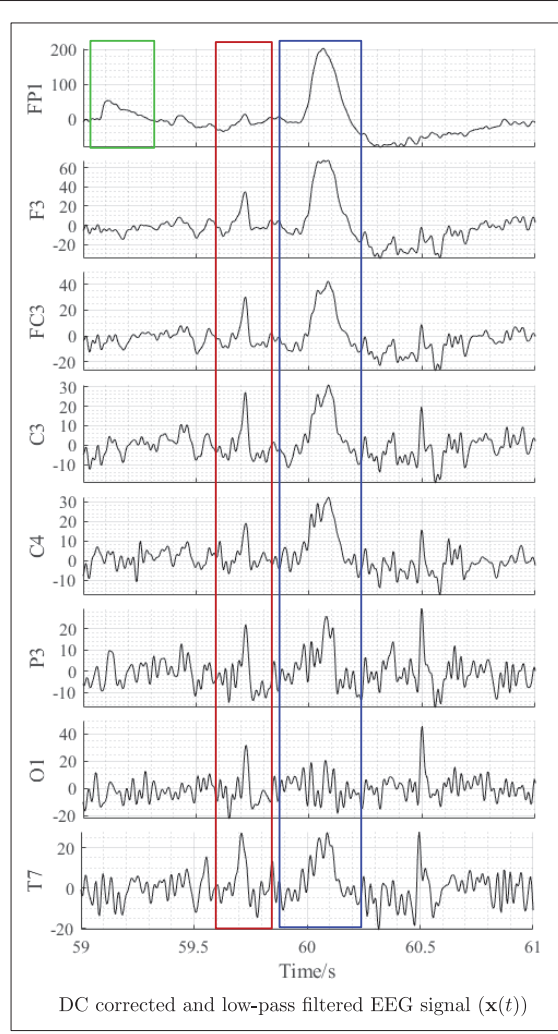

$\mathbf{u}(\mathrm{t})=\mathbf{W} \mathbf{x}(\mathrm{t})$

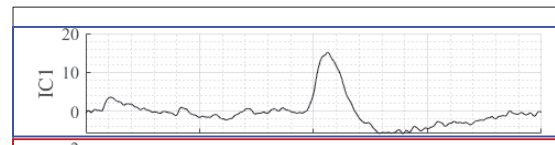

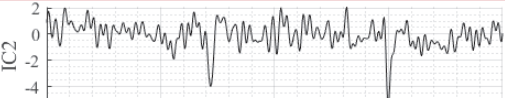

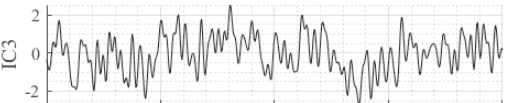

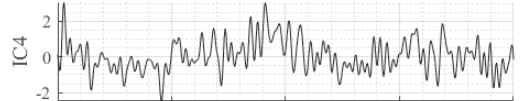

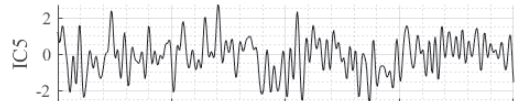

4
0
0
-2

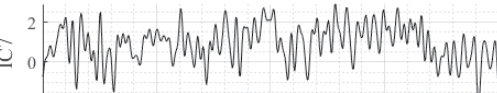

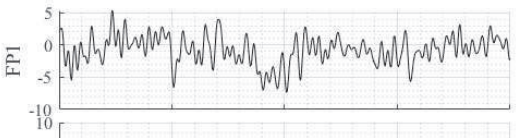

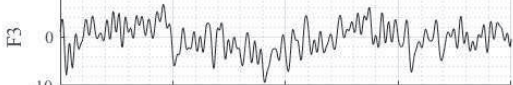

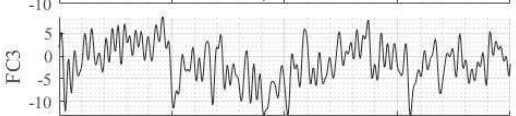

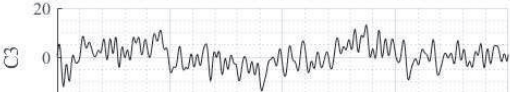

10.

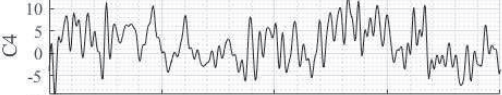

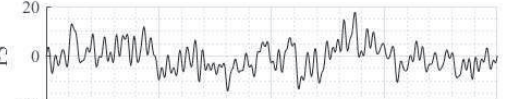

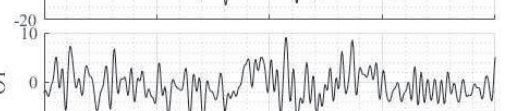

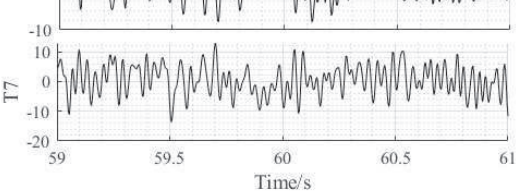

Artifact removed EEG signal $(\hat{\mathbf{x}}(t))$

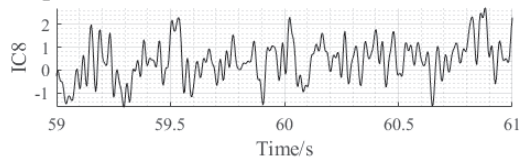

Time courses of the Independent Components (ICs) $(\mathrm{u}(\mathrm{t}))$

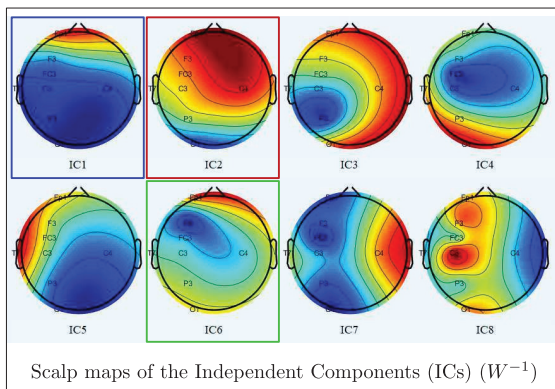

$\hat{\mathbf{x}}(t)=W^{-1} \hat{\mathbf{u}}(t)$

FIGURE 5 | Illustration of EEG artifactual components identification using ICA for one participant. The red, blue, and green rectangles correspond to cardiac activity, eye blink, and eye movement artifacts, respectively. All data were referenced to the right earlobe (A2). 
TABLE 2 | Significant EEG spectral feature modulations and the corresponding electrode locations following the gross motor interaction with HapticMASTER.

\begin{tabular}{|c|c|c|c|c|c|c|c|c|}
\hline \multirow{2}{*}{ Spectral feature } & \multirow{2}{*}{ Electrode location } & \multicolumn{2}{|c|}{ Sample mean \pm std } & \multicolumn{4}{|c|}{ Paired samples $t$-test } & \multirow{2}{*}{ Direction of change } \\
\hline & & Baseline & Recovery & $t$ & $d f$ & $p$-value & $r$ & \\
\hline \multirow[t]{2}{*}{$\delta_{\text {relative }}$} & C3 & $0.542 \pm 0.109$ & $0.476 \pm 0.067$ & 2.593 & 9 & 0.029 & 0.654 & $\downarrow$ \\
\hline & FC3 & $0.180 \pm 0.068$ & $0.225 \pm 0.069$ & -2.378 & 9 & 0.041 & 0.621 & $\uparrow$ \\
\hline \multirow[t]{2}{*}{$\alpha_{\text {relative }}$} & C3 & $0.198 \pm 0.070$ & $0.259 \pm 0.095$ & -3.148 & 9 & 0.012 & 0.724 & $\uparrow$ \\
\hline & P3 & $0.271 \pm 0.094$ & $0.330 \pm 0.154$ & -2.646 & 9 & 0.027 & 0.661 & $\uparrow$ \\
\hline$\frac{(\theta+\alpha)}{\beta}$ & C3 & $8.151 \pm 4.349$ & $8.923 \pm 4.167$ & -2.787 & 9 & 0.021 & 0.681 & $\uparrow$ \\
\hline$\frac{\alpha}{\beta}$ & C3 & $4.213 \pm 2.612$ & $4.997 \pm 2.812$ & -2.403 & 9 & 0.040 & 0.625 & $\uparrow$ \\
\hline
\end{tabular}

$\uparrow=$ significant increase. $\downarrow=$ significant decrease.
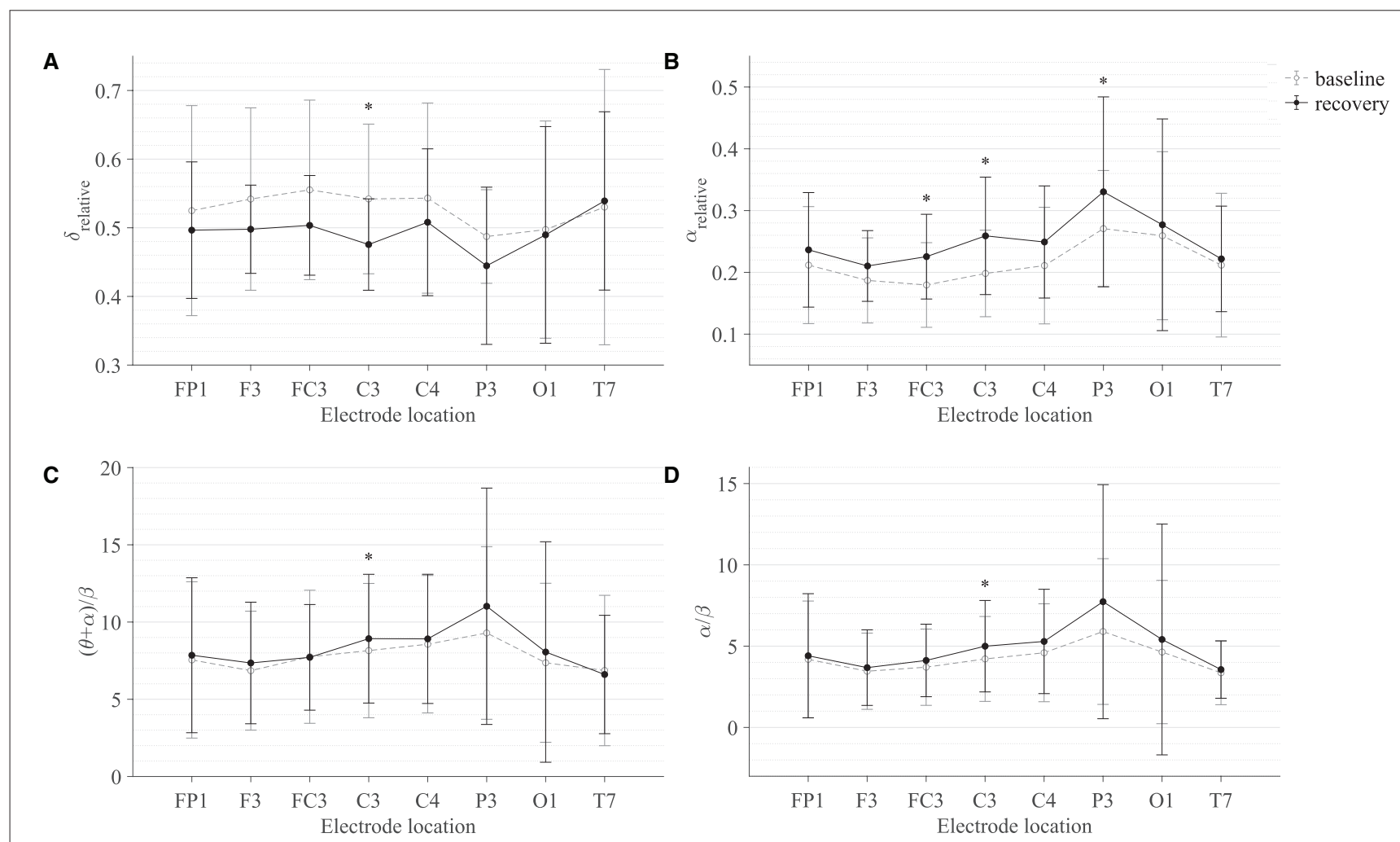

FIGURE 6 | Comparison of the sample mean and standard deviation of (A) $\delta_{\text {relative }}$, (B) $\alpha_{\text {relative }}$, (C) $(\theta+\alpha) / \beta$, and (D) $\alpha / \beta$ of all participants between baseline and recovery states for the gross motor interaction with HapticMASTER. The statistical significance is represented by an asterisk: i.e., ${ }^{*} \mathrm{p}<0.05$.

fatigue induced by the gross movements may have altered the EEG activity around the left central and left parietal regions.

\subsection{Modulations in EEG Spectral Features Following the Robot-Mediated Fine Motor Interaction With SCRIPT Passive Orthosis}

Table 3 summarises the paired-samples $t$-test results of the statistically significant EEG spectral feature modulations following the fine motor interaction with SCRIPT passive orthosis. Figure 8 shows the sample mean and standard deviation of the substantive EEG spectral features during baseline and recovery states. An increase of $\theta_{\text {relative }}$ and $\alpha_{\text {relative }}$ is visible in both Figures $\mathbf{8 B}, \mathbf{C}$ on all electrodes. A significant increase in $\alpha_{\text {relative }}$ is visible on FP1 $(t=-2.871, p=0.018, r$ $=0.691)$ and C3 $(t=-2.555, p=0.031, r=0.648)$ electrodes, whereas the significant difference in $\theta_{\text {relative }}$ is on the $\mathrm{C} 4$ electrode ( $t=-3.507, p=0.007, r=0.760)$. The effect of these significant variations in $\alpha_{\text {relative }}$ and $\theta_{\text {relative }}$ are also of larger magnitude, thereby suggesting that these variations are substantive findings. In contrast, a general decrease in $\delta_{\text {relative }}$ on all electrodes and a significant decrease on the FP1 electrode with a larger effect size $(t=3.066, p=0.013, r=0.715)$ can be found in Figure 8A. No significant differences were visible in $\beta_{\text {relative }}$ and ratio band power measures. In general, these results show that the fatigue induced by fine motor interactions alters not only the activities 
A

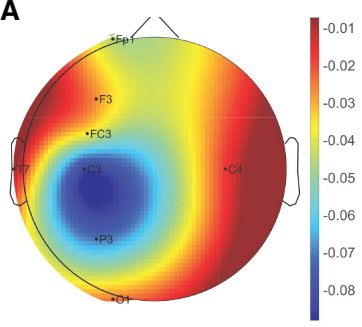

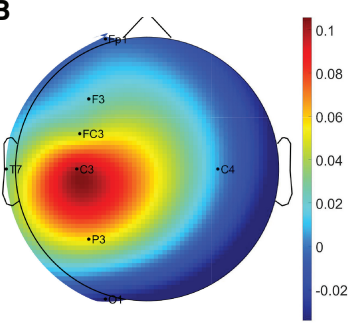

C

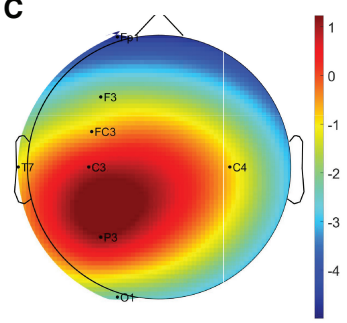

D

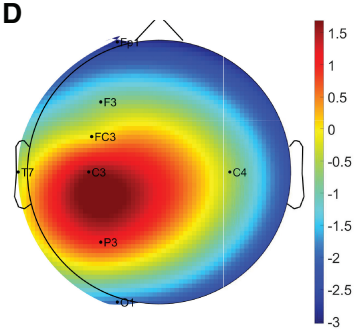

FIGURE 7 | Brain topographies for the difference between recovery and baseline states (i.e., difference $=$ recovery - baseline) of $(\mathbf{A}) \delta_{\text {relative }}$ (B) $\alpha_{\text {relative }},(\mathbf{C})(\theta+\alpha) / \beta$, and (D) $\alpha / \beta$ for one participant following the gross motor interaction with HapticMASTER. In each brain map, the nose is represented by the triangle on the top, and the right hemisphere is on the right. For $\alpha_{\text {relative }},(\theta+\alpha) / \beta$, and $\alpha / \beta$, the red-shaded areas indicate a larger increase whereas the blue-shaded areas indicate a decrease. For $\delta_{\text {relative }}$, the blue-shaded areas indicate a larger decrease whereas the red-shaded areas indicate a smaller decrease.

around the sensorimotor cortex but also the frontopolar cortex. Figure 9 shows the brain topographies of the difference between recovery and baseline states (i.e., difference = recovery - baseline) of the substantive EEG features for one participant who reported a greater increase in mental fatigue than physical fatigue following the fine motor task. Topographical distributions also show that the variations in the EEG features around frontopolar and central brain regions may have been caused by the fatigue that resulted from the robot-mediated fine motor interaction.

\subsection{Subjective Measures of Fatigue Level and Workload}

Most participants who performed the robot-mediated gross motor interaction with HapticMASTER reported increased physical fatigue following the task. Six participants showed a greater change in physical fatigue scores than in mental fatigue scores, and two participants showed an equal rise in both physical and mental fatigue scores. Therefore, the subjective ratings suggest that the gross motor interaction may have induced physical fatigue. In contrast, most participants who performed the fine motor task reported that their mental fatigue was increased following the robotic interaction. Four participants showed a greater change in mental fatigue scores than physical fatigue scores, and two participants showed an equal rise in both physical and mental fatigue scores. Therefore, the subjective ratings suggest that the fine motor interaction, on the other hand, may have induced mental fatigue. Furthermore, most participants reported that the gross motor task was more physically demanding than mentally demanding. In contrast, most participants revealed that the fine motor task required greater mental demand or equally physical and mental demand. A comparison of the subjective measures of physical and mental fatigue levels before and after the robot-mediated gross and fine motor interactions and the physical and mental workload of the two tasks is shown in Figure 10.

Figure 11 shows the association between the variations in fatigue levels and the rated workload following the robotmediated gross and fine motor interactions. All participants who experienced a greater increase in physical fatigue than the change in mental fatigue following the gross motor task also rated that the underlying physical workload of the gross motor task was greater than the mental workload. All participants who experienced a greater increase in mental fatigue than physical fatigue following the fine motor task rated that the fine motor task required a greater mental demand than the physical demand. The gross motor task involves the movement and coordination of proximal joints and muscles of the upper limb (shoulder and arm) to control the robot arm between target points. The fine motor task requires considerable attention and decision-making skills combined with hand and finger movements to catch the fish when it reaches the seashell. Therefore, the subjective responses imply that the gross motor task performed with HapticMASTER may have greatly contributed to the development of physical fatigue due to the increased physical demand. In contrast, the fine motor task performed with SCRIPT passive orthosis may have mainly induced mental fatigue due to the increased mental demand required during the task.

\subsection{Association of Changes in Fatigue Level With the Substantive EEG Feature Modulations}

Most participants who reported an increase in physical fatigue following the robot-mediated gross motor interaction also showed a greater increase in $\alpha_{\text {relative }}$ on FC3, C3, and P3 electrodes, $(\theta+\alpha) / \beta$ and $\alpha / \beta$ on the $\mathrm{C} 3$ electrode in comparison to the participants who reported no change or reduction in physical fatigue. Similarly, a greater decrease in $\delta_{\text {relative }}$ on the C3 electrode was also found in most participants who experienced increased physical fatigue. Therefore, the above findings show that the significant changes in $\delta_{\text {relative }}, \alpha_{\text {relative }},(\theta+\alpha) / \beta$ and $\alpha / \beta$ around the motor cortex are likely related to the physical fatigue accumulated following the gross motor task. All six participants who reported increased mental fatigue following the robot-mediated fine motor interaction showed a decrease in $\delta_{\text {relative }}$ on the FP1 electrode. Five participants also showed an increase in $\alpha_{\text {relative }}$ on FP1 and C3 electrodes, and four 
TABLE 3 | Significant EEG spectral feature modulations and the corresponding electrode locations following the fine motor interaction with SCRIPT passive orthosis.

\begin{tabular}{|c|c|c|c|c|c|c|c|c|}
\hline \multirow{2}{*}{ Spectral feature } & \multirow{2}{*}{ Electrode location } & \multicolumn{2}{|c|}{ Sample mean \pm std } & \multicolumn{4}{|c|}{ Paired samples $t$-test } & \multirow{2}{*}{ Direction of change } \\
\hline & & Baseline & Recovery & $t$ & $d f$ & $p$-value & $r$ & \\
\hline$\delta_{\text {relative }}$ & FP1 & $0.550 \pm 0.096$ & $0.504 \pm 0.106$ & 3.066 & 9 & 0.013 & 0.715 & $\downarrow$ \\
\hline$\theta_{\text {relative }}$ & $\mathrm{C} 4$ & $0.193 \pm 0.033$ & $0.226 \pm 0.039$ & -3.507 & 9 & 0.007 & 0.760 & $\uparrow$ \\
\hline \multirow[t]{2}{*}{$\alpha_{\text {relative }}$} & FP1 & $0.179 \pm 0.075$ & $0.211 \pm 0.104$ & -2.871 & 9 & 0.018 & 0.691 & $\uparrow$ \\
\hline & C3 & $0.202 \pm 0.127$ & $0.227 \pm 0.117$ & -2.555 & 9 & 0.031 & 0.648 & $\uparrow$ \\
\hline
\end{tabular}

$\uparrow=$ significant increase. $\downarrow$ = significant decrease.
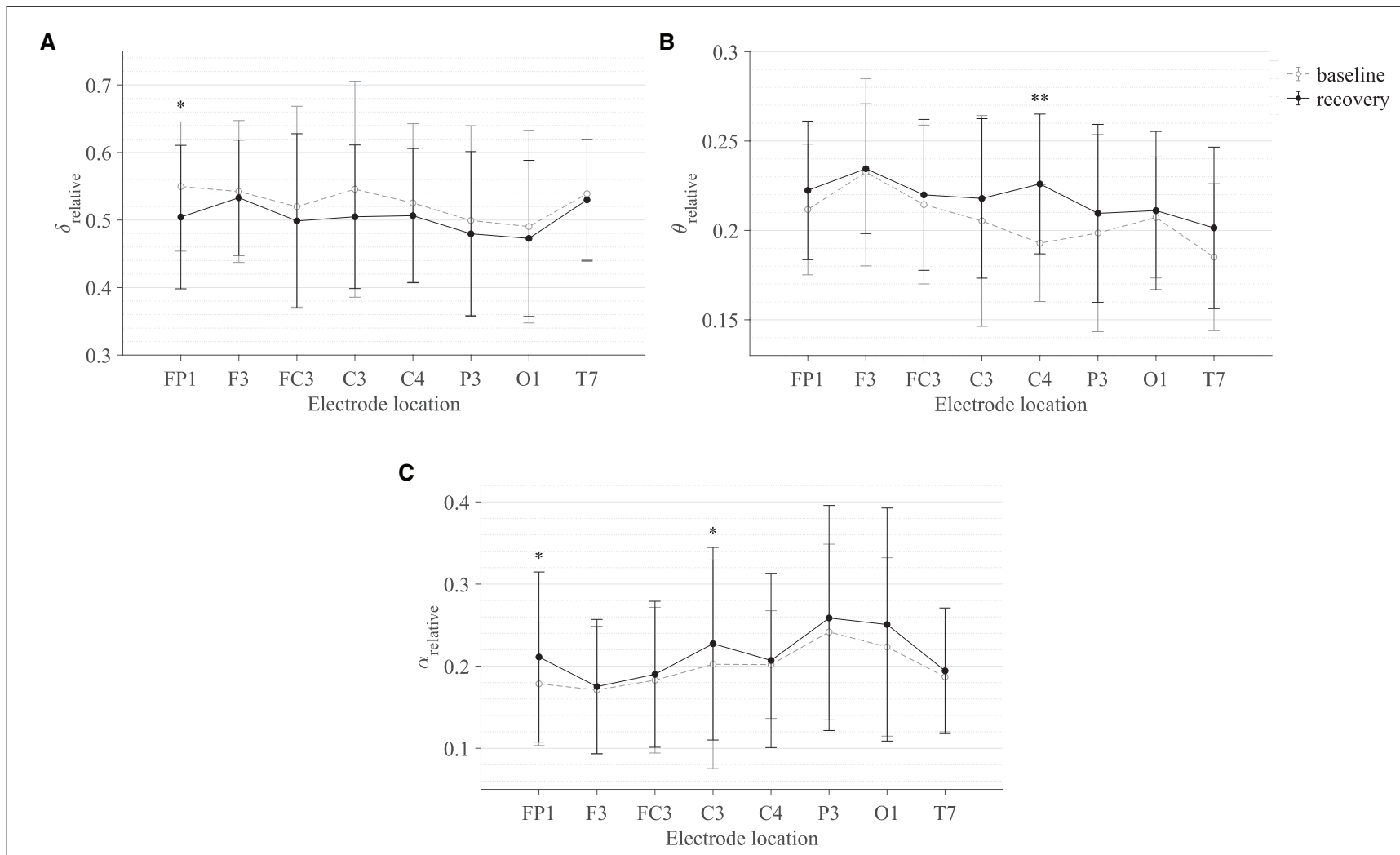

FIGURE 8 | Comparison of the sample mean and standard deviation of (A) $\delta_{\text {relative }}$ (B) $\theta_{\text {relative, and }}$ (C) $\alpha_{\text {relative }}$ of all participants between baseline and recovery states for the fine motor interaction with SCRIPT passive orthosis. The statistical significance is represented by an asterisk: i.e., ${ }^{*} p<0.05$ and ${ }^{* \star} p<0.01$.

participants showed an increase in $\theta_{\text {relative }}$ on C4. Therefore, the modulations in EEG spectral features around the prefrontal cortex presumably reflect increased mental fatigue following the robot-mediated fine movements. Figure 12 shows the association between the modulations in $\delta_{\text {relative }}$ and $\alpha_{\text {relative }}$ and the variations in subjective measures of physical and mental fatigue levels following the robot-mediated gross and fine motor tasks.

\section{DISCUSSION}

This preliminary experiment investigated cortical-related changes associated with fatigue in upper limb robot-mediated gross and fine motor interactions. The findings of this experiment indicate that it is possible to monitor fatigue induced by robotmediated interactions using EEG spectral features, which can have further utility for robot-mediated post-stroke therapy.
The most prominent finding was a significant increase in the $\alpha_{\text {relative }}$ following both the robot-mediated gross and fine motor interactions. It is known that $\alpha$ activity is most commonly visible during relaxed conditions and decreased attention levels. Also, in drowsy but wakeful states when increased efforts are taken to maintain the level of attention and alertness, increased $\alpha$ activity is visible (Klimesch, 1999). In contrast, when an individual is in an alert state, suppression of $\alpha$ activity is visible. Task-related desynchronisation, which leads to a decrease in $\alpha$ activity, can be interpreted as an electrophysiological correlate of increased activation of the cortical areas (excited neural structures) that produce motor behaviour or process sensory or cognitive information (Pfurtscheller et al., 1996; Pfurtscheller, 1997). Therefore, the increased $\alpha_{\text {relative }}$ following the robotmediated interactions may reflect decreased cortical activity and a reduced capacity for information processing in the underlying 

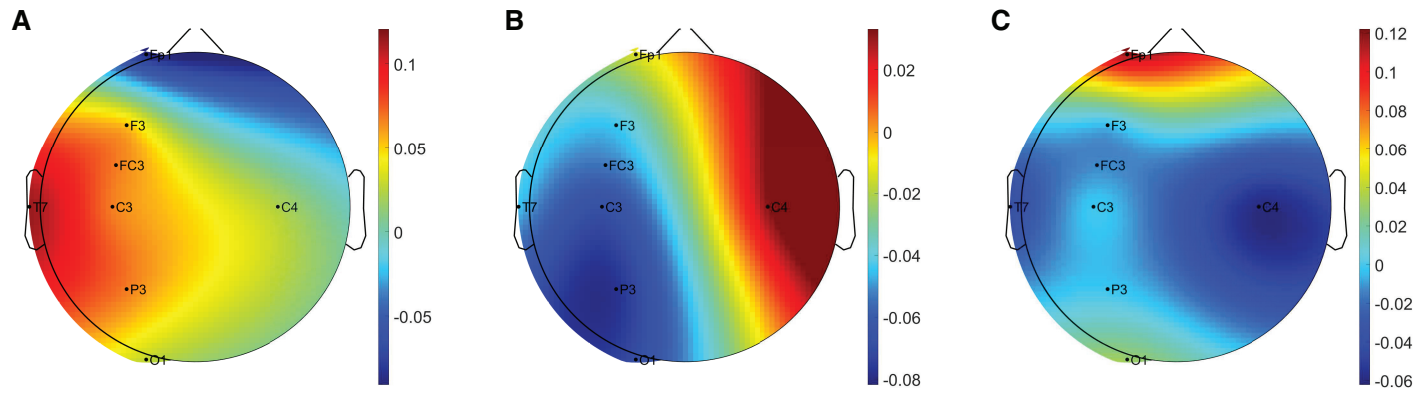

FIGURE 9 | Brain topographies for the difference between recovery and baseline states (i.e., difference $=$ recovery - baseline) of (A) $\delta_{\text {relative }}$, (B) $\theta_{\text {relative }}$, and (C) $\alpha_{\text {relative }}$ for one participant following the fine motor interaction with SCRIPT passive orthosis. In each brain map, the nose is represented by the triangle on the top, and the right hemisphere is on the right. The red-shaded areas indicate a larger increase whereas the blue-shaded areas indicate a larger decrease.

A

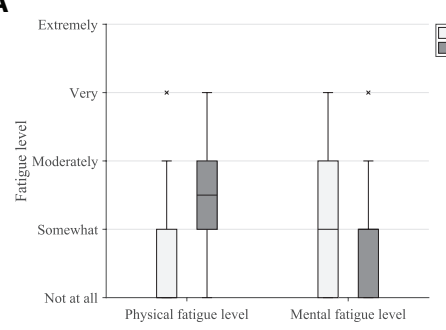

B

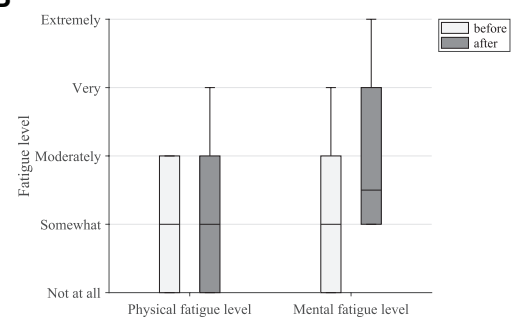

C

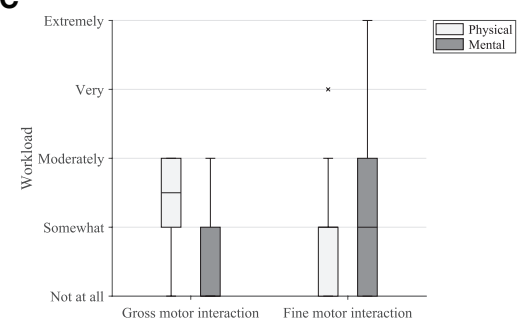

FIGURE 10 | Subjective measures of fatigue level and workload. (A) Comparison of the subjective measures of fatigue level before and after the gross motor interaction with HapticMATER. (B) Comparison of the subjective measures of fatigue level before and after the fine motor interaction with SCRIPT passive orthosis. (C) Comparison of the subjective measures of physical and mental workload following the gross and fine motor interactions.
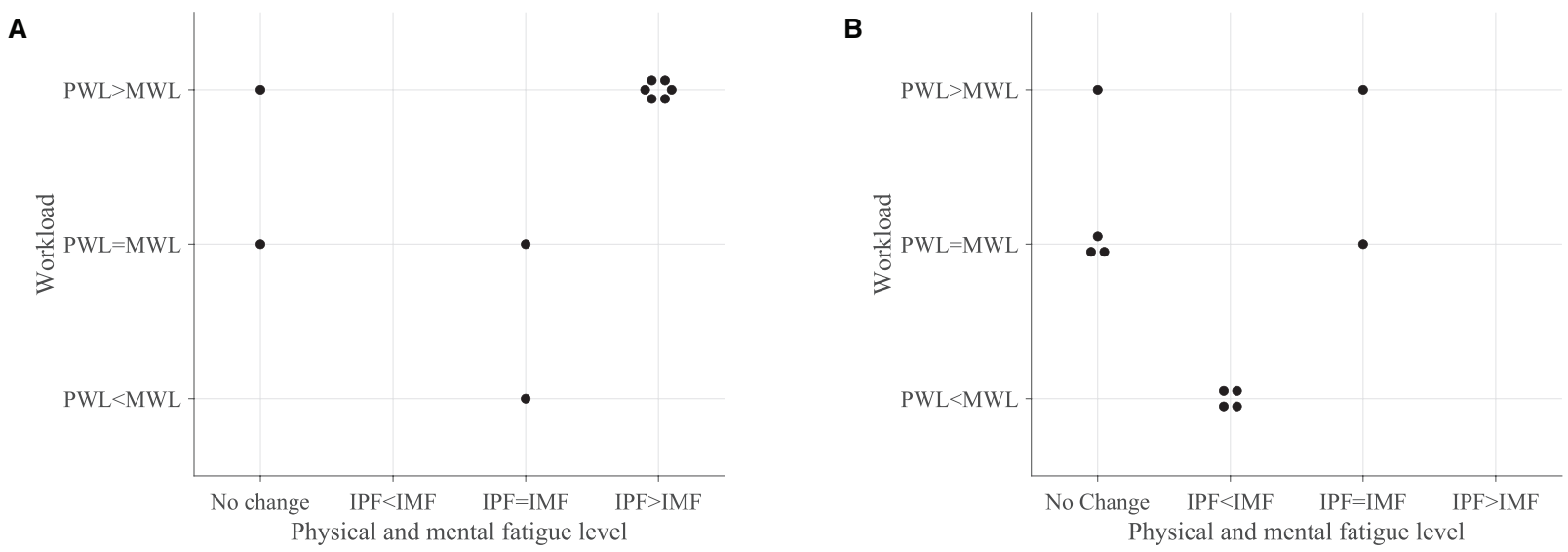

FIGURE 11 | Association between the variations in fatigue levels and the rated workload following the (A) robot-mediated gross motor interaction with HapticMASTER and (B) robot-mediated fine motor interaction with SCRIPT passive orthosis. The "IPF" and "IMF" refers to the increase in physical and mental fatigue scores following the robot-mediated interactions, respectively. No change refers to no increase or a decrease in both fatigue levels. The "PWL" and "MWL" refers to the rated physical and mental workload, respectively.

cortical regions due to fatigue. This finding is in agreement with the findings of previous fatigue studies (Eoh et al., 2005; Barwick et al., 2012; Zhao et al., 2012; Fan et al., 2015; Zou et al.,
2015). Thus, we suggest that the observed modulations in $\alpha_{\text {relative }}$ presumably reflect the changes in an individual's fatigue level following upper limb robot-mediated interactions. Furthermore, 
Gross motor interaction with HapticMASTER

A

$\delta_{\text {relative }}$

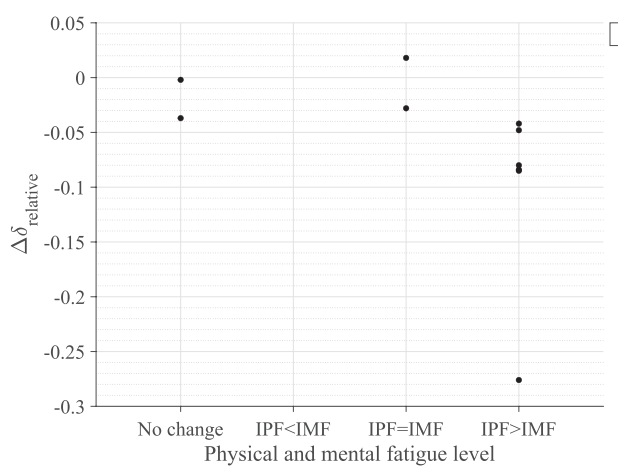

B

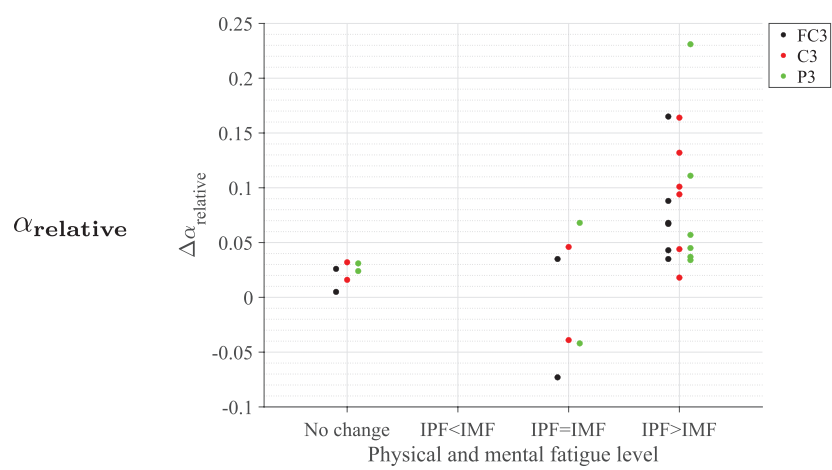

Fine motor interaction with SCRIPT passive orthosis
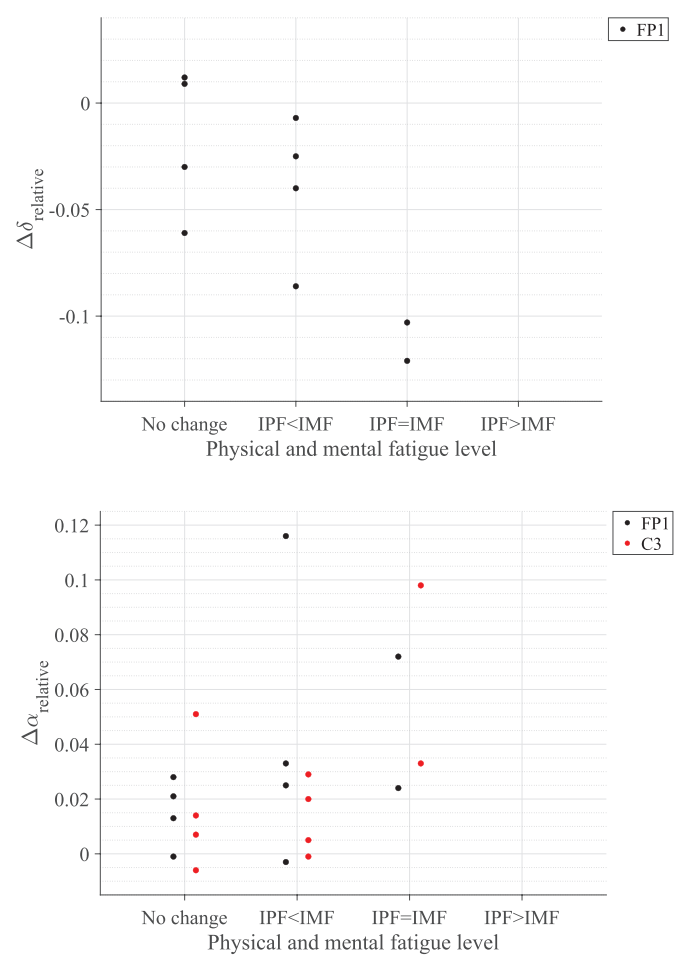

FIGURE 12 | Comparison of the modulations in $\delta_{\text {relative }}$ and $\alpha_{\text {relative }}$ with the variations in fatigue levels following the (A) robot-mediated gross motor interaction with HapticMASTER and (B) robot-mediated fine motor interaction with SCRIPT passive orthosis. The $\Delta$ represents the difference in each EEG feature following the robot-mediated interactions (i.e., recovery - baseline). The "IPF" and "IMF" refers to the increase in physical and mental fatigue scores following the robot-mediated interactions, respectively. No change refers to no increase or a decrease in both fatigue levels.

the above inference was also supported by the participants' feedback on the changes in their physical and mental fatigue levels after the assigned task; thereby suggesting that $\alpha_{\text {relative }}$ is a reliable EEG-based fatigue index that can be used to monitor fatigue accumulated during human-robot interactions.

Topographical differences found in the prominent EEG spectral features indicate that the brain regions most affected by fatigue may depend on the physical and mental workload associated with the task and the differences in the usage of the proximal and distal upper arm. In the gross motor interaction, participants were instructed to move the HapticMASTER robot arm in a linear trajectory to reach the two target points visible in the virtual reality environment. In a visually guided reaching task, the sensory system extracts spatial information about the target, and a movement plan is created and executed by the motor cortex (Sabes, 2000; Gevins and Smith, 2007). The premotor cortex, primary somatosensory cortex, and posterior parietal cortex integrate motor and sensory information for planning and coordinating complex movements. Also, HapticMASTER is an end-effector based robot, and the proximal upper limbs (arm and shoulder) are predominantly used when moving the robot arm between target points during the gross motor task. Therefore, the significant rise in $\alpha_{\text {relative }}$ found at FC3, C3, and P3 electrode locations presumably reflects the inhibition of premotor cortex, primary somatosensory cortex, and posterior parietal cortex due to physical fatigue accumulated during the arm reach/return task. A previous study has also shown that the upper limb reaching tasks performed using the HapticMASTER induced muscle fatigue (Thacham Poyil et al., 2020a,b). Conversely, in the fine motor task, participants were expected to perform hand open/close gestures only when a fish was near the seashell in the virtual environment. Therefore, the fine motor task required more sustained attention and decision-making than the gross motor task. Laureiro-Martínez et al. (2014) also found that a stronger activation in the frontopolar cortex is associated with higher decision-making efficiency. In addition, active movements consisting of repetitive opening and closing of the hand are shown to activate the contralateral primary sensorimotor cortex (Guzzetta et al., 2007). Therefore, the increased $\alpha_{\text {relative }}$ over FP1 and $\mathrm{C} 3$ electrodes following the repetitive fine movements appear to reflect an altered decision-making efficiency of an individual, in addition to the deactivation in the motor cortex associated with fatigue. The topographical variations in $\alpha_{\text {relative }}$ were also supported by the participants' feedback on their fatigue level after each interaction. The greater changes in $\alpha_{\text {relative }}$ following the gross motor task were also associated with a greater increase 
in physical fatigue. In contrast, the greater changes in $\alpha_{\text {relative }}$ following the fine motor task were associated with a greater increase in mental fatigue or an equal increase in both physical and mental fatigue levels.

It has been established in the literature that EEG activity shifts from high frequencies toward slower waves with the progression of fatigue; thus, the ratio between low-frequency and highfrequency power can also be considered as a reliable measure of fatigue (Eoh et al., 2005; Jap et al., 2009). This experiment found significant differences only in $(\theta+\alpha) / \beta$ and $\alpha / \beta$ on the $\mathrm{C} 3$ electrode following the physically fatiguing gross motor task. These findings were also supported by the participants' feedback on their fatigue level. Furthermore, there were no significant differences in the power ratios due to the fine motor task. Although the significant changes on the C3 electrode were only visible for $\alpha_{\text {relative }}$, a slight increase in $\theta_{\text {relative }}$ and a decrease in $\beta_{\text {relative }}$ were also found after the gross motor task. Therefore, the findings suggest that gross motor interaction increased the low-frequency activities while suppressing the high-frequency activities on the C3 electrode, which may have caused the significant increase of $(\theta+\alpha) / \beta$ and $\alpha / \beta$. Eoh et al. (2005), Jap et al. (2009), Chen et al. (2013), Fan et al. (2015) also reported a significant rise in both $(\theta+\alpha) / \beta$ and $\alpha / \beta$ with fatigue.

The suppression in $\delta_{\text {relative }}$ following the robot-mediated interactions is contrary to some previous studies that have suggested a statistically significant increase or no significant difference in $\delta$ activities due to fatigue (Lal and Craig, 2002; Craig et al., 2012; Zhao et al., 2012). However, a non-significant reduction in $\delta_{\text {relative }}$ around all brain regions after a simulated driving task was reported in Zhao et al. (2012). In this experiment, a significant decrease in $\delta_{\text {relative }}$ was found on the C3 electrode following the gross motor task and on the FP1 electrode following the fine motor task. Most participants who reported an increase in their physical fatigue level after the robotmediated gross motor task also have experienced a decrease in $\delta_{\text {relative }}$ on the C3 electrode. Similarly, all participants who reported an increase in their mental fatigue level following the robot-mediated fine motor task also showed a decrease in $\delta_{\text {relative }}$. Therefore, the subjective measures of fatigue level support the suppression in $\delta_{\text {relative }}$ due to fatigue build-up and the topographical variations found in the two tasks. The methodological differences of the previous studies could explain these discrepancies as these studies were based on vehicle driving tasks, whereas our experiment was focused on gross and fine motor tasks in a human-robot interaction scenario. Harmony et al. (1996) proposed that increased attention to internal processing (i.e., "internal concentration") during mental tasks might cause an increase in the delta activity. In order to accurately perform the two tasks in this experiment, higher concentration and attention levels are essential. Therefore, the reduction in $\delta_{\text {relative }}$ associated with the robotic interactions may suggest deficient inhibitory control and information-processing mechanisms. This finding, while preliminary, suggests that fatigue may have negatively affected an individual's attention and internal concentration levels. Therefore, $\delta_{\text {relative could }}$ also be used as an EEG-based measure of fatigue in robotmediated interactions.
The ipsilateral primary somatosensory cortex is also shown to increase its level of activation during prolonged sustained and intermittent sub-maximal muscle contractions to compensate for fatigue (Liu et al., 2003). In this experiment, a significant change in the $\mathrm{C} 4$ electrode was visible only for $\theta_{\text {relative }}$ following the fine motor task. Theta oscillations in EEG have shown to be prominent during cognitive processing that requires higher mental effort and is positively related to task difficulty (Gevins et al., 1997). Barwick et al. (2012), Cheng and Hsu (2011), and Zhao et al. (2012) also reported an increase in $\theta_{\text {relative }}$ due to fatigue build-up. Therefore, the rise in $\theta_{\text {relative }}$ on $\mathrm{C} 4$ may reflect the fatigue-related changes in the ipsilateral brain activation caused by the fine motor task.

The spatial precision of the EEG recordings taken in this experiment was limited since the EEG data acquisition system could only support eight electrode locations. Furthermore, only a limited number of participants were tested, and each participant interacted with only one robotic interface. Therefore, future research should examine more electrode locations and consider a cross-over study design with a higher sample size where each participant is exposed to both fatiguing robotic interactions.

\section{CONCLUSION}

This paper investigates the modulations in EEG spectral features associated with fatigue induced by robot-mediated upper limb gross and fine motor interactions. It was found that the fatigue induced by the gross movements mostly altered the EEG activity around the central and parietal brain regions, whereas the fine movements mostly altered the EEG activity around the frontopolar and central brain regions. These regional differences in significant EEG spectral features are most likely due to the differences in the nature of the task (fine/gross motor and distal/proximal upper limb) that may have differently altered the physical and mental fatigue level of an individual. We have shown that EEG correlates of fatigue progressed during robotmediated interactions are specific to the physical or cognitive nature of the task performed using the proximal or distal upper limb. Further studies will explore whether the specificity is due to the difference in the motor skills considered (fine/gross motor) or the usage of upper limbs (distal/proximal upper limb). Given that fatigue during robot-mediated therapy can be estimated via EEG spectral features, we believe that the findings could potentially be used to moderate the level of fatigue during post-stroke rehabilitation, acknowledging that stroke patients are more likely to be fatigued than healthy individuals. Moreover, it would be possible to derive more personalised robot-mediated post-stroke rehabilitation regimes that would utilise the individual fatigue levels as a tool to increase the efficacy of upper limb robotmediated rehabilitation.

\section{DATA AVAILABILITY STATEMENT}

The datasets presented in this article are not readily available because ethical consent to share the data publicly alongside publications was not sought at data collection, and therefore 
it is not possible to share the data. Requests to access the datasets should be directed to Farshid Amirabdollahian, f.amirabdollahian2@herts.ac.uk.

\section{ETHICS STATEMENT}

The studies involving human participants were reviewed and approved by Science and Technology Ethics Committee with Delegated Authority of the University of Hertfordshire. The patients/participants provided their written informed consent to participate in this study.

\section{AUTHOR CONTRIBUTIONS}

UD carried out the data collection, analysis, and wrote the first draft of the manuscript. FA and VS obtained the research funding and supervised the research. All authors contributed to the

\section{REFERENCES}

Alderman, R. B. (1965). Influence of local fatigue on speed and accuracy in motor learning. Res. Quart. Amer. Assoc. Health Phys. Educ. Recreat. 36, 131-140. doi: 10.1080/10671188.1965.10614670

American Clinical Neurophysiology Society. (2006). Guideline 5: guidelines for standard electrode position nomenclature. J. Clin. Neurophysiol. 23, 107-110. doi: 10.1097/00004691-200604000-00006

Amirabdollahian, F., Ates, S., Basteris, A., Cesario, A., Buurke, J., Hermens, H., et al. (2014). Design, development and deployment of a hand/wrist exoskeleton for home-based rehabilitation after stroke - SCRIPT project. Robotica 32, 1331-1346. doi: 10.1017/S02635747140 02288

Amirabdollahian, F., Loureiro, R., Gradwell, E., Collin, C., Harwin, W., and Johnson, G. (2007). Multivariate analysis of the Fugl-Meyer outcome measures assessing the effectiveness of GENTLE/S robot-mediated stroke therapy. J. NeuroEng. Rehabil. 4, 4. doi: 10.1186/1743-0003-4-4

Bailey, S. P., Hall, E. E., Folger, S. E., and Miller, P. C. (2008). Changes in EEG during graded exercise on a recumbent cycle ergometer. J. Sports Sci. Med. 7, 505-11.

Barwick, F., Arnett, P., and Slobounov, S. (2012). EEG correlates of fatigue during administration of a neuropsychological test battery. Clin. Neurophysiol. 123, 278-284. doi: 10.1016/j.clinph.2011.06.027

Baumeister, J., Reinecke, K., Schubert, M., Schade, J., and Weiss, M. (2012). Effects of induced fatigue on brain activity during sensorimotor control. Eur. J. Appl. Physiol. 112, 2475-2482. doi: 10.1007/s00421-011-2215-6

Borghini, G., Astolfi, L., Vecchiato, G., Mattia, D., and Babiloni, F. (2014). Measuring neurophysiological signals in aircraft pilots and car drivers for the assessment of mental workload, fatigue and drowsiness. Neurosci. Biobehav. Rev. 44, 58-75. doi: 10.1016/j.neubiorev.2012.10.003

Branscheidt, M., Kassavetis, P., Anaya, M., Rogers, D., Huang, H. D., Lindquist, M. A., et al. (2019). Fatigue induces long-lasting detrimental changes in motorskill learning. eLife 8, e40578. doi: 10.7554/eLife.40578

Caldwell, J. A., Hall, K. K., and Erickson, B. S. (2002). EEG data collected from helicopter pilots in flight are sufficiently sensitive to detect increased fatigue from sleep deprivation. Int. J. Aviation Psychol. 12, 19-32. doi: 10.1207/S15327108IJAP1201_3

Cardoso, J. F. (1998). Blind signal separation: statistical principles. Proc. IEEE 86, 2009-2025. doi: 10.1109/5.720250

Cardoso, J. F., and Souloumiac, A. (1993). "Blind beamforming for non-gaussian signals," in IEE Proceedings F (Radar and Signal Processing), Vol. 140 (IET), $362-370$.

Carron, A. (1972). Motor performance and learning under physical fatigue. Med. Sci. Sports 4, 101-106. conception and design of the experiment, manuscript revision, and approved the submitted version.

\section{FUNDING}

This work was supported by the Ph.D studentship awarded to UD from the University of Hertfordshire, United Kingdom.

\section{ACKNOWLEDGMENTS}

This experiment was conducted as part of the Ph.D programme pursued by UD at the University of Hertfordshire, United Kingdom. The authors would like to thank all the participants who contributed their time and effort to this experiment. A preprint of this paper is available on bioRxiv (Dissanayake et al., 2021).

Carron, A. V. (1969). Physical fatigue and motor learning. Res. Quart. Amer. Assoc. Health Phys. Educ. Recreat. 40, 682-686. doi: 10.1080/10671188.1969.10614902

Chemuturi, R., Amirabdollahian, F., and Dautenhahn, K. (2013). Adaptive training algorithm for robot-assisted upper-arm rehabilitation, applicable to individualised and therapeutic human-robot interaction. J. Neuroeng. Rehabil. 10, 102. doi: 10.1186/1743-0003-10-102

Chen, C., Li, K., Wu, Q., Wang, H., Qian, Z., and Sudlow, G. (2013). EEG-based detection and evaluation of fatigue caused by watching 3DTV. Displays 34, 81-88. doi: 10.1016/j.displa.2013.01.002

Cheng, S. Y., and Hsu, H. T. (2011). "Mental fatigue measurement using EEG," in Risk Management Trends, ed G. Nota (IntechOpen). doi: 10.5772/16376

Cotten, D. J., Thomas, J. R., Spieth, W. R., and Biasiotto, J. (1972). Temporary fatigue effects in a gross motor skill. J. Motor Behav. 4, 217-222.

Cowley, J. C., and Gates, D. H. (2017). Proximal and distal muscle fatigue differentially affect movement coordination. PLoS ONE 12, e0172835. doi: 10.1371/journal.pone.0172835

Craig, A., Tran, Y., Wijesuriya, N., and Nguyen, H. (2012). Regional brain wave activity changes associated with fatigue. Psychophysiology 49, 574-582. doi: 10.1111/j.1469-8986.2011.01329.x

Debener, S., Thorne, J., Schneider, T. R., and Viola, F. C. (2010). "Using ICA for the analysis of multi-channel EEG data," in Simultaneous EEG and fMRI: Recording, Analysis, and Application, eds M. Ullsperger, and D. Stefan (New York, NY: Oxford University Press), 121-133. doi: 10.1093/acprof:oso/9780195372731.003.0008

Delorme, A., Sejnowski, T., and Makeig, S. (2007). Enhanced detection of artifacts in EEG data using higher-order statistics and independent component analysis. Neuroimage 34, 1443-1449. doi: 10.1016/j.neuroimage.2006.11.004

Dissanayake, U. C. (2021). Assessment of Fatigue in Robot-Mediated Upper Limb Interactions Using EEG. (Hatfield: University of Hertfordshire).

Dissanayake, U. C., Steuber, V., and Amirabdollahian, F. (2021). EEG spectral feature modulations associated with fatigue in robot-mediated upper limb gross motor and fine motor interactions. bioRxiv. doi: 10.1101/2021.04.22.440968

Eoh, H. J., Chung, M. K., and Kim, S. H. (2005). Electroencephalographic study of drowsiness in simulated driving with sleep deprivation. Int. J. Ind. Ergon. 35, 307-320. doi: 10.1016/j.ergon.2004.09.006

Fan, X., Zhou, Q., Liu, Z., and Xie, F. (2015). Electroencephalogram assessment of mental fatigue in visual search. Bio Med. Mater. Eng. 26, S1455-S1463. doi: 10.3233/BME-151444

Foong, R., Ang, K. K., Quek, C., Guan, C., Phua, K. S., Kuah, C. W. K., et al. (2019). Assessment of the efficacy of EEG-based MI-BCI with visual feedback and EEG correlates of mental fatigue for upper-limb stroke rehabilitation. IEEE Trans. Biomed. Eng. 67, 786-795. doi: 10.1109/TBME.2019.2921198

g.tec medical engineering GmbH (2014a). g.GAMMACap2 EEG sensor cap: Instructions for use V2.14.00. Austria: g.tec medical engineering $\mathrm{GmbH}$. 
g.tec medical engineering GmbH (2014b). g.GAMMAsys Active Electrode System: Instructions for use V2.14.01. Austria: g.tec medical engineering $\mathrm{GmbH}$.

Gevins, A., and Smith, M. E. (2007). "Electroencephalography (EEG) in neuroergonomics," in Neuroergonomics: The brain at Work, eds R. Parasuraman and M. Rizzo (New York, NY: Oxford University Press), 15-31.

Gevins, A., Smith, M. E., McEvoy, L., and Yu, D. (1997). High-resolution EEG mapping of cortical activation related to working memory: effects of task difficulty, type of processing, and practice. Cereb. Cortex 7, 374-385. doi: 10.1093/cercor/7.4.374

Gibson, H., and Edwards, R. (1985). Muscular exercise and fatigue. Sports Med. 2, 120-132. doi: 10.2165/00007256-198502020-00004

Godwin, M. A., and Schmidt, R. A. (1971). Muscular fatigue and learning a discrete motor skill. Res. Quart. Amer. Assoc. Health Phys. Educ. Recreat. 42, 374-382. doi: 10.1080/10671188.1971.10615084

Guzzetta, A., Staudt, M., Petacchi, E., Ehlers, J., Erb, M., Wilke, M., et al. (2007). Brain representation of active and passive hand movements in children. Pediatric Res. 61, 485-490. doi: 10.1203/pdr.0b013e3180332c2e

Harmony, T., Fernández, T., Silva, J., Bernal, J., Díaz-Comas, L., Reyes, A., et al. (1996). EEG delta activity: an indicator of attention to internal processing during performance of mental tasks. Int. J. Psychophysiol. 24, 161-171. doi: 10.1016/S0167-8760(96)00053-0

Jap, B. T., Lal, S., Fischer, P., and Bekiaris, E. (2009). Using EEG spectral components to assess algorithms for detecting fatigue. Exp. Syst. Appl. 36, 2352-2359. doi: 10.1016/j.eswa.2007.12.043

Jung, T.P., Makeig, S., Humphries, C., Lee, T.W., Mckeown, M. J., Iragui, V., et al. (2000). Removing electroencephalographic artifacts by blind source separation. Psychophysiology 37, 163-178. doi: 10.1111/1469-8986.3720163

Jung, T. P., Humphries, C., Lee, T. W., Makeig, S., McKeown, M. J., Iragui, V., et al. (1998). "Extended ICA removes artifacts from electroencephalographic recordings," in Advances in Neural Information Processing Systems (Cambridge, MA: MIT Press), 894-900.

Käthner, I., Wriessnegger, S. C., Müller-Putz, G. R., Kübler, A., and Halder, S. (2014). Effects of mental workload and fatigue on the P300, alpha and theta band power during operation of an ERP (P300) brain-computer interface. Biol. Psychol. 102, 118-129. doi: 10.1016/j.biopsycho.2014.07.014

Klimesch, W. (1999). EEG alpha and theta oscillations reflect cognitive and memory performance: a review and analysis. Brain Res. Rev. 29, 169-195. doi: 10.1016/S0165-0173(98)00056-3

Lal, S. K., and Craig, A. (2002). Driver fatigue: electroencephalography and psychological assessment. Psychophysiology 39, 313-321. doi: $10.1017 /$ S0048577201393095

Lal, S. K. L., and Craig, A. (2001). A critical review of the psychophysiology of driver fatigue. Biol. Psychol. 55, 173-194. doi: 10.1016/S0301-0511(00)00085-5

Laureiro-Martínez, D., Canessa, N., Brusoni, S., Zollo, M., Hare, T., Alemanno, F., et al. (2014). Frontopolar cortex and decision-making efficiency: comparing brain activity of experts with different professional background during an exploration-exploitation task. Front. Human Neurosci. 7:927. doi: $10.3389 /$ fnhum.2013.00927

Lerdal, A., Bakken, L. N., Kouwenhoven, S. E., Pedersen, G., Kirkevold, M., Finset, A., et al. (2009). Poststroke fatigue - a review. J. Pain Symptom Manage. 38, 928-949. doi: 10.1016/j.jpainsymman.2009.04.028

Liu, J. Z., Shan, Z. Y., Zhang, L. D., Sahgal, V., Brown, R. W., and Yue, G. H. (2003). Human brain activation during sustained and intermittent submaximal fatigue muscle contractions: an fMRI study. J. Neurophysiol. 90, 300-312. doi: $10.1152 /$ jn. 00821.2002

Lorist, M. M., Boksem, M. A., and Ridderinkhof, K. R. (2005). Impaired cognitive control and reduced cingulate activity during mental fatigue. Cogn. Brain Res. 24, 199-205. doi: 10.1016/j.cogbrainres.2005.01.018

Makeig, S., Bell, A. J., Jung, T. P., and Sejnowski, T. J. (1996). "Independent component analysis of electroencephalographic data," in Advances in Neural Information Processing Systems (Cambridge, MA: MIT Press), 145-151.

Marcora, S. M., Staiano, W., and Manning, V. (2009). Mental fatigue impairs physical performance in humans. J. Appl. Physiol. 106, 857-864. doi: 10.1152/japplphysiol.91324.2008

Massar, S. A., Wester, A. E., Volkerts, E. R., and Kenemans, J. L. (2010). Manipulation specific effects of mental fatigue: evidence from novelty processing and simulated driving. Psychophysiology 47, 1119-1126. doi: $10.1111 / j .1469-8986.2010 .01028 . x$
Mehta, R. K., and Parasuraman, R. (2014). Effects of mental fatigue on the development of physical fatigue: a neuroergonomic approach. Hum. Factors 56 , 645-656. doi: 10.1177/0018720813507279

Ng, S. C., and Raveendran, P. (2007). "EEG peak alpha frequency as an indicator for physical fatigue," in 11th Mediterranean Conference on Medical and Biomedical Engineering and Computing 2007, (Berlin: Springer), 517-520.

Pfurtscheller, G. (1997). EEG event-related desynchronization (ERD) and synchronization (ERS). Electroencephalograph. Clin. Neurophysiol. 1, 26.

Pfurtscheller, G., Stancak, A. Jr., and Neuper, C. (1996). Event-related synchronization (ERS) in the alpha band - an electrophysiological correlate of cortical idling: a review. Int. J. Psychophysiol. 24, 39-46. doi: 10.1016/S0167-8760(96)00066-9

Pinegger, A., Wriessnegger, S. C., Faller, J., and Müller-Putz, G. R. (2016). Evaluation of different EEG acquisition systems concerning their suitability for building a brain-computer interface: case studies. Front. Neurosci. 10:441. doi: $10.3389 /$ fnins.2016.00441

Prasad, G., Herman, P., Coyle, D., McDonough, S., and Crosbie, J. (2010). Applying a brain-computer interface to support motor imagery practice in people with stroke for upper limb recovery: a feasibility study. J. Neuroeng. Rehabil. 7, 60. doi: $10.1186 / 1743-0003-7-60$

Sabes, P. N. (2000). The planning and control of reaching movements. Curr. Opin. Neurobiol. 10, 740-746. doi: 10.1016/S0959-4388(00)00149-5

Staub, F., and Bogousslavsky, J. (2001). Fatigue after stroke: a major but neglected issue. Cerebrovascul. Dis. 12, 75-81. doi: 10.1159/000047685

Sterr, A., and Furlan, L. (2015). A case to be made: theoretical and empirical arguments for the need to consider fatigue in post-stroke motor rehabilitation. Neural Regener. Res. 10, 1195-1197. doi: 10.4103/1673-5374.162689

Tanaka, M., Shigihara, Y., Ishii, A., Funakura, M., Kanai, E., and Watanabe, Y. (2012). Effect of mental fatigue on the central nervous system: an electroencephalography study. Behav. Brain Funct. 8, 48. doi: 10.1186/1744-9081-8-48

Thacham Poyil, A., Steuber, V., and Amirabdollahian, F. (2020a). Adaptive robot mediated upper limb training using electromyogram-based muscle fatigue indicators. PLOS ONE 15, e0233545. doi: 10.1371/journal.pone.0233545

Thacham Poyil, A. T., Steuber, V., and Amirabdollahian, F. (2020b). Influence of muscle fatigue on electromyogram - kinematic correlation during robot-assisted upper limb training. J. Rehabil. Assist. Technol. Eng. 7, 2055668320903014. doi: 10.1177/2055668320903014

Thomas, J. R., Cotten, D. J., Spieth, W. R., and Abraham, N. L. (1975). Effects of fatigue on stabilometer performance and learning of males and females. Med. Sci. Sports 7, 203-206. doi: 10.1249/00005768-197500730-00018

Tran, Y., Craig, A., Craig, R., Chai, R., and Nguyen, H. (2020). The influence of mental fatigue on brain activity: evidence from a systematic review with meta-analyses. Psychophysiology 57, e13554. doi: 10.1111/psyp.13554

Tran, Y., Wijesuryia, N., Thuraisingham, R. A., Craig, A., and Nguyen, H. T. (2008). "Increase in regularity and decrease in variability seen in electroencephalography (EEG) signals from alert to fatigue during a driving simulated task," in 2008 30th Annu. Int. Conf. IEEE Eng. Med. Biol. Soc. (IEEE) (Vancouver), 1096-1099.

Trejo, L. J., Kubitz, K., Rosipal, R., Kochavi, R. L., and Montgomery, L. D. (2015). EEG-based estimation and classification of mental fatigue. Psychology 6, 572. doi: 10.4236/psych.2015.65055

Van Cutsem, J., Marcora, S., De Pauw, K., Bailey, S., Meeusen, R., and Roelands, B. (2017). The effects of mental fatigue on physical performance: a systematic review. Sports Med. 47, 1569-1588. doi: 10.1007/s40279-016-0672-0

Wang, Y., Cao, L., Hao, D., Rong, Y., Yang, L., Zhang, S., et al. (2017). Effects of force load, muscle fatigue and extremely low frequency magnetic stimulation on EEG signals during side arm lateral raise task. Physiol. Meas. 38, 745. doi: 10.1088/1361-6579/aa6b4b

Welch, P. (1967). The use of fast fourier transform for the estimation of power spectra: a method based on time averaging over short, modified periodograms. IEEE Trans. Audio Electroacoust. 15, 70-73. doi: 10.1109/TAU.1967.1161901

Williams, J., and Singer, R. N. (1975). Muscular fatigue and the learning and performance of a motor control task. J. Motor Behav. 7, 265-269. doi: 10.1080/00222895.1975.10735044

Xu, R., Zhang, C., He, F., Zhao, X., Qi, H., Zhou, P., et al. (2018). How physical activities affect mental fatigue based on EEG energy, connectivity, and complexity. Front. Neurol. 9:915. doi: 10.3389/fneur.2018.00915 
Yao, B., Liu, J. Z., Brown, R. W., Sahgal, V., and Yue, G. H. (2009). Nonlinear features of surface EEG showing systematic brain signal adaptations with muscle force and fatigue. Brain Res. 1272, 89-98. doi: 10.1016/j.brainres.2009.03.042

Zhao, C., Zhao, M., Liu, J., and Zheng, C. (2012). Electroencephalogram and electrocardiograph assessment of mental fatigue in a driving simulator. Accident Anal. Prevent. 45, 83-90. doi: 10.1016/j.aap.2011.11.019

Zou, B., Liu, Y., Guo, M., and Wang, Y. (2015). EEG-based assessment of stereoscopic $3 \mathrm{D}$ visual fatigue caused by vergence-accommodation conflict. J. Display Technol. 11, 1076-1083. doi: 10.1109/JDT.2015.2451087

Conflict of Interest: The authors declare that the research was conducted in the absence of any commercial or financial relationships that could be construed as a potential conflict of interest.
Publisher's Note: All claims expressed in this article are solely those of the authors and do not necessarily represent those of their affiliated organizations, or those of the publisher, the editors and the reviewers. Any product that may be evaluated in this article, or claim that may be made by its manufacturer, is not guaranteed or endorsed by the publisher.

Copyright $(2022$ Dissanayake, Steuber and Amirabdollahian. This is an open-access article distributed under the terms of the Creative Commons Attribution License (CC $B Y)$. The use, distribution or reproduction in other forums is permitted, provided the original author(s) and the copyright owner(s) are credited and that the original publication in this journal is cited, in accordance with accepted academic practice. No use, distribution or reproduction is permitted which does not comply with these terms. 